\title{
On the Power of Unambiguity in Alternating Machines
}

\author{
Holger Spakowski* \\ Institut für Informatik \\ Heinrich-Heine-Universität Düsseldorf \\ 40225 Düsseldorf, Germany \\ spakowsk@cs. uni-duesseldorf .de
}

\author{
Rahul Tripathi ${ }^{\dagger}$ \\ Department of Computer Science \\ University of Rochester \\ Rochester, NY 14627, USA \\ rahult@cs.rochester. edu
}

Technical Report URCS-TR-2004-851

October 07, 2004

\begin{abstract}
Recently, the property of unambiguity in alternating Turing machines has received considerable attention in the context of analyzing globally-unique games by Aida et al. [ACRW04] and in the design of efficient protocols involving globally-unique games by Crâsmaru et al. [CGRS04]. This paper investigates the power of unambiguity in alternating Turing machines in the following settings:

1. We construct a relativized world in which unambiguity based hierarchies-AUPH, $\mathrm{UPH}$, and $\mathcal{U P H}$ - are infinite. We construct another relativized world where UAP (unambiguous alternating polynomial-time) is not contained in the polynomial hierarchy.

2. We define the bounded-level unambiguous alternating solution class $\operatorname{UAS}(k)$, for every $k \geq 1$, as the class of sets for which strings in the set are accepted unambiguously by some polynomial-time alternating Turing machine $N$ with at most $k$ alternations, while strings not in the set either are rejected or are accepted with ambiguity by $N$. We construct a relativized world where for all $k \geq 1$, $\mathrm{UP}_{\leq k} \subset \mathrm{UP}_{\leq k+1}$ and $\mathrm{UAS}(k) \subset \mathrm{UAS}(k+1)$.
\end{abstract}

3. Finally, we show that robustly $k$-level unambiguous alternating Turing machines accept languages that are computable in $\mathrm{P}^{\Sigma_{k}^{p} \oplus \mathcal{A}}$, for every oracle $\mathcal{A}$. This generalizes a result of Hartmanis and Hemachandra [HH90].

Keywords: structural complexity, unambiguous computation, alternation, relativization.

\section{Introduction}

Chandra, Kozen, and Stockmeyer [CKS81] introduced the notion of alternation as a generalization of nondeterminism: Alternation allows switching of existential

\footnotetext{
${ }^{*}$ Research supported in part by a grant from the DAAD and by DFG project RO 1202/9-1.

${ }^{\dagger}$ Supported in part by grant NSF-CCF-0426761.
} 
and universal quantifiers, whereas nondeterminism allows only existential quantifiers throughout the computation. Alternation has proved to be a central notion in complexity theory. For instance, the polynomial hierarchy has a characterization in terms of bounded-level alternation [Sto77,CKS81], the complexity class PSPACE can be characterized in terms of polynomial length-bounded alternation [CKS81], and many important classes have characterizations based on variants of alternation (see Chapter 19 of [Pap94]).

Unambiguity in nondeterministic computation is related to issues such as worstcase cryptography and the closure properties of \#P (the class of functions that count the number of accepting paths of NP machines). The complexity class UP captures the notion of unambiguity in nondeterministic polynomial-time Turing machines. It is known that one-to-one one-way functions exist if and only if $\mathrm{P} \neq \mathrm{UP}[\mathrm{Ko85}$,GS88] and that UP equals probabilistic polynomial-time if and only if \#P is closed under every polynomial-time computable operation [OH93]. Factoring, a natural problem with cryptographic applications, belongs to UP $\cap$ coUP and is not known to belong to a subclass of UP $\cap$ coUP nontrivially.

This paper studies the power of unambiguity in alternating computations. Niedermeier and Rossmanith [NR98] gave the following definition of unambiguity in alternating Turing machines: An alternating Turing machine is unambiguous if every accepting existential configuration has exactly one move to an accepting configuration and every rejecting universal configuration has exactly one move to a rejecting configuration. They introduced a natural analog UAP (unambiguous alternating polynomial-time) of UP for alternating Turing machines. Lange and Rossmanith [LR94] proposed three different approaches to define a hierarchy for unambiguous computations: The alternating unambiguous polynomial hierarchy AUPH, the unambiguous polynomial hierarchy UPH, and the promise unambiguous hierarchy $\mathcal{U P H}$. Though it is known that Few $\subseteq \mathrm{UAP} \subseteq \mathrm{SPP}[\mathrm{NR} 98]$ and AUPH $\subseteq$ $\mathrm{UPH} \subseteq \mathcal{U P H} \subseteq \mathrm{UAP}[$ LR94,CGRS04], a number of questions- such as, whether UAP is contained in the polynomial hierarchy, whether the unambiguity based hierarchies intertwine, whether these hierarchies are infinite, or whether some hierarchy is contained in a fixed level of the other hierarchy-related to these hierarchies have remained open [LR94]. Relatedly, Hemaspaandra and Rothe [HR97] showed that the existence of sparse Turing complete sets for UP has consequences on the structure of unambiguity based hierarchies.

Recently, Aida et al. [ACRW04] introduced "uniqueness" properties for two-player games of perfect information such as Checker, Chess, and Go. A two-person perfect information game has the global uniqueness property if every winning position of player 1 has a unique move to win and every mis-step by player 1 is punishable by a unique winning reply by player 2 throughout the course of the game. Aida et al. [ACRW04] showed that the class of languages that reduce to globally-unique games, i.e., games with global uniqueness property, is the same as the class UAP. In another recent paper, Crâsmaru et al. [CGRS04] designed a protocol by which a series of globallyunique games can be combined into a single globally-unique game, even under the condition that the result of the new game is a non-monotone function of the results of the individual games that are unknown to the players. In complexity theoretic terms, they showed that the class UAP is self-low, i.e., UAP ${ }^{\mathrm{UAP}}=\mathrm{UAP}$. They also observed that the graph isomorphism problem, whose membership in SPP was shown by Arvind and Kurur [AK02], in fact belongs to the subclass UAP of SPP. 
In this paper, we investigate the power of unambiguity based alternating computation in three different settings. First, we construct a relativized world in which the unambiguity based hierarchies-AUPH, UPH, and $\mathcal{U P H}$ - are infinite. This extends the separation of the relativized polynomial hierarchy [Yao85,Hås87] to the separations of the unambiguity based relativized hierarchies. We construct another relativized world where UAP is not contained in the polynomial hierarchy. This latter oracle result strengthens a result (relative to an oracle, UAP differs from the second level of $\mathcal{U P H}$ ) of Crâsmaru et al. [CGRS04]. Our results show that proving that any of the unambiguity based hierarchies is finite or that UAP is contained in the polynomial hierarchy is impossible by relativizable proof techniques. We mention that the structure of relativized hierarchies of classes has been investigated extensively in complexity theory (see, for instance [Yao85,Hås87, $\mathrm{CGH}^{+} 89, \mathrm{Ko} 89, \mathrm{Ko}$ 91]) and our investigation is a work in this direction.

Second, for every $k \geq 1$, we define a complexity class $\operatorname{UAS}(k)$ as the class of sets for which every string in the set is accepted unambiguously by some polynomial-time alternating Turing machine $N$ with at most $k$ alternations, while strings not in the set either are rejected or are accepted with ambiguity by $N$. A variant of this class (denoted by UAS in this paper), where the number of alternations is allowed to be unbounded, was studied by Wagner [Wag92] as the class $\nabla \mathrm{P}$ of all sets which can be accepted by polynomial-time alternating Turing machines using partially defined AND and OR functions. ${ }^{1}$ Beigel [Bei89] defined the class $\mathrm{UP}_{\leq k(n)}$ as the class of sets in NP that are accepted by nondeterministic polynomial-time Turing machines with at most $k(n)$ accepting paths on each input of length $n$. Beigel [Bei89] constructed an oracle $\mathcal{A}$ such that $\mathrm{P}^{\mathcal{A}} \subset \mathrm{UP}^{\mathcal{A}} \subset \mathrm{UP}_{k(n)}^{\mathcal{A}} \subset \mathrm{UP}_{k(n)+1}^{\mathcal{A}} \subset \mathrm{FewP}^{\mathcal{A}} \subset \mathrm{NP}^{\mathcal{A}}$, for every polynomial $k(n) \geq 2$. We show that there is a relativized world $\mathcal{B}$ such that, for all $k \geq 1, \mathrm{UP}_{\leq k}^{\mathcal{B}} \subset \mathrm{UP}_{\leq k+1}^{\mathcal{B}}, \mathrm{UAS}(k)^{\mathcal{B}} \subset \mathrm{UAS}(k+1)^{\mathcal{B}}$, and relative to $\mathcal{B}$, the second level of $\mathcal{U P H}$ is not contained in any level of AUPH.

Finally, we investigate the power of polynomial-time alternating Turing machines that preserve the bounded-level unambiguity property for every oracle. We show that a polynomial-time alternating Turing machine that preserves $k$-level alternation unambiguously in every relativized world requires only weak oracle access in every relativized world, i.e., for every oracle $\mathcal{A}$, the language of such a machine can be computed in $\mathrm{P}^{\Sigma_{k}^{p} \oplus \mathcal{A}}$. This is a generalization of a result of Hartmanis and Hemachandra [HH90], which states that if a nondeterministic polynomial-time Turing machine is robustly categorical (i.e., for no oracle and for no input, the machine has more than one accepting path), then for every oracle $\mathcal{A}$, the machine accepts a language in $\mathrm{P}^{\mathrm{NP} \oplus \mathcal{A}}$.

\section{Preliminaries}

Let $\mathbb{N}^{+}$denote the set of positive integers. We assume without loss of generality that the root of a computation tree of every alternating Turing machine (or, ATM in short) is an existential node. For any deterministic or nondeterministic, or alternating Turing machine $N, A \subseteq \Sigma^{*}$, and $x \in \Sigma^{*}$, we use the shorthand $N^{A}(x)$ for "the computation of

\footnotetext{
${ }^{1}$ The partial counterparts $\mathrm{AND}^{*}$ and $\mathrm{OR}^{*}$ differ from boolean functions AND and OR, respectively, as follows: $\mathrm{AND}^{*}$ is undefined for input $(0,0)$ and $\mathrm{OR}^{*}$ is undefined for input $(1,1)$. Thus, these partially defined boolean functions are the unambiguous counterparts of boolean AND and OR functions, respectively.
} 
$N$ with oracle $A$ on input $x$," the shorthand ctree $(N, A, x)$ for "the computation tree of $N^{A}(x)$," and for every node $u$ in ctree $(N, A, x)$, the shorthand $\operatorname{subtree}(N, A, x, u)$ for "the computation subtree of ctree $(N, A, x)$ rooted at $u$." We recursively assign levels in a computation tree $T$ of an ATM as follows: (a) the root of $T$ is at level 1, (b) if a node $v$ is assigned a level $i$ and if $v$ is an existential node, then the first nonexistential (i.e., universal or leaf) node $w$ reachable along some path from $v$ to a leaf node of $T$ is assigned level $i+1,(\mathrm{c})$ if a node $v$ is assigned a level $i$ and if $v$ is a universal node, then the first nonuniversal (i.e., existential or leaf) node $w$ reachable along some path from $v$ to a leaf node of $T$ is assigned level $i+1$, and (d) for all other nodes in $T$, the concept of levels is insignificant to this work and so the levels are undefined. We term the nonleaf nodes for which levels are defined as the salient nodes in the computation tree of an ATM. For any salient node or leaf node $\vartheta$, we use level $(\vartheta)$ to denote the level of $\vartheta$ in the computation tree of an ATM. For any $k \in \mathbb{N}^{+}$, a $k$-level ATM is one for which, on any input, the maximum level assigned to a salient node in the computation tree of the ATM is at most $k$. For every ATM $N, A \subseteq \Sigma^{*}, x \in \Sigma^{*}$, the switch time of $\operatorname{ctree}(N, A, x)$ is the maximum number of computation steps required to reach from a salient node $v$ at level $i$ to a node $w$ at level $i+1$, where $w$ is in subtree $(N, A, x, v)$ and the maximum is over all $v, w$, and $i$. A normalized ATM is one in which, for every oracle $A$, input $x$, level $i$ in ctree $(N, A, x)$, and salient node $v$ at level $i$, the number of computation steps required to reach from $v$ to a node $w$ at level $i+1$, where $w$ is in subtree $(N, A, x, v)$, equals the switch time of the ATM.

We say that a computation tree or subtree $T$ of an ATM is unambiguous if every accepting existential node has exactly one move to an accepting node and every rejecting universal node has exactly one move to a rejecting node. If a computation tree or subtree $T$ is unambiguous and if $T$ accepts (rejects), then we say that $T$ accepts (respectively, rejects) with unambiguity. For every $\operatorname{ATM} N, A \subseteq \Sigma^{*}$, and $x \in \Sigma^{*}$, we say that $N^{A}(x)$ accepts (rejects) with unambiguity if $N^{A}(x)$ accepts (respectively, rejects) and $\operatorname{ctree}(N, A, x)$ is unambiguous. If, for every $x \in \Sigma^{*}, \operatorname{ctree}(N, A, x)$ is unambiguous, then we say that $N$ with oracle $A$ (or, simply $N$, if $A=\emptyset$ ) is unambiguous.

For every polynomial $p($.$) and for every predicate R(x, y, z)$ of variables $x, y, z$, we use $\left(\exists^{p} ! y\right)\left(\forall^{p} ! z\right) R(x, y, z)$ to indicate that there exists a unique value $y_{1}$ for the $y$ variable with $\left|y_{1}\right| \leq p(|x|)$ such that for all values $z_{1}$ for the $z$ variable with $\left|z_{1}\right| \leq p(|x|), R\left(x, y_{1}, z_{1}\right)$ is true, and for all values $y_{2} \neq y_{1}$ for the $y$ variable with $\left|y_{2}\right| \leq p(|x|)$, there exists a unique value $z\left(y_{2}\right)$ for the $z$ variable with $\left|z\left(y_{2}\right)\right| \leq p(|x|)$, such that $R\left(x, y_{2}, z\left(y_{2}\right)\right)$ is false. In the same way, we interpret expressions, such as $\left(\exists^{p} ! y_{1}\right)\left(\forall^{p} ! y_{2}\right)\left(\exists^{p} ! y_{3}\right) \ldots R\left(x, y_{1}, y_{2}, y_{3}, \ldots\right)$, with an arbitrary number of unambiguous alternations.

Definition 2.1 (Unambiguity Based Hierarchies [LR94,NR98]) 1. The alternating unambiguous polynomial hierarchy AUPH $=_{d f} \bigcup_{k \geq 0} \mathrm{AU} \Sigma_{k}^{p}$, where $\mathrm{AU} \Sigma_{0}^{p}={ }_{d f} \mathrm{P}$ and for every $k \geq 1, \mathrm{AU} \Sigma_{k}^{p}$ is the class of all sets $L \subseteq \Sigma^{*}$ for which there exist a polynomial $p($.$) and a polynomial-time computable predicate R$ such that, for all $x \in \Sigma^{*}$,

$$
\begin{aligned}
& x \in L \quad \Longrightarrow\left(\exists^{p} ! y_{1}\right)\left(\forall^{p} ! y_{2}\right) \ldots\left(Q^{p} ! y_{k}\right) R\left(x, y_{1}, y_{2}, \ldots, y_{k}\right), \text { and } \\
& x \notin L
\end{aligned}
$$

where $Q=\exists$ and $\bar{Q}=\forall$ if $k$ is odd, and $Q=\forall$ and $\bar{Q}=\exists$ if $k$ is even. 
2. The unambiguous polynomial hierarchy is $\mathrm{UPH}={ }_{d f} \bigcup_{k \geq 0} \mathrm{U} \Sigma_{k}^{p}$, where $\mathrm{U} \Sigma_{0}^{p}={ }_{d f} \mathrm{P}$ and for every $k \geq 1, \mathrm{U} \Sigma_{k}^{p}={ }_{d f} \mathrm{UP}^{\mathrm{U} \Sigma_{k-1}^{p}}$.

3. The promise unambiguous polynomial hierarchy is $\mathcal{U P H}=_{d f} \bigcup_{k>0} \mathcal{U} \Sigma_{k}^{p}$, where $\mathcal{U} \Sigma_{0}^{p}=_{d f} \mathrm{P}, \mathcal{U} \Sigma_{1}^{p}=d_{d f} \mathrm{UP}$, and for every $k \geq 2, \mathcal{U} \Sigma_{k}^{p}$ is the class of all sets $L \in \Sigma_{k}^{p}$ such that for some oracle $\operatorname{NPTM} s N_{1}, \ldots, N_{k}, L=L\left(N_{1}^{L\left(N_{2}{ }^{. L\left(N_{k}\right)}\right.}\right)$, and for every $x \in \Sigma^{*}$ and for every $1 \leq i \leq k-1$, if $N_{i}$ asks a query $w$ to its oracle $L\left(N_{i+1}^{. L\left(N_{k}\right)}\right)$ during the computation of $N_{i}^{.{ }^{. L\left(N_{k}\right)}}(x)$, then $N_{i+1}^{. L\left(N_{k}\right)}(w)$ has at most one accepting path.

Definition 2.2 [NR98] UAP is the class of sets accepted by unambiguous ATMs in polynomial time.

Theorem 2.3 1. For all $k \geq 0, \mathrm{AU} \Sigma_{k}^{p} \subseteq \mathrm{U} \Sigma_{k}^{p} \subseteq \mathcal{U} \Sigma_{k}^{p} \subseteq \Sigma_{k}^{p}$ [LR94].

2. For all $k \geq 1, \mathrm{UP}_{\leq k} \subseteq \mathrm{AU} \Sigma_{k}^{p} \subseteq \mathrm{U} \Sigma_{k}^{p} \subseteq \mathcal{U} \Sigma_{k}^{p} \subseteq \mathrm{UAP}$ ([LR94] + [CGRS04]).

3. Few $\subseteq \mathrm{UAP} \subseteq \mathrm{SPP}([\mathrm{LR} 94]+[\mathrm{NR} 98])$.

\section{Relativized Separations of Unambiguity Based Hierarchies}

In this section, we apply random restrictions of circuits for separating the levels of unambiguity based hierarchies. Sheu and Long [SL96] constructed an oracle $\mathcal{A}$ relative to which UP contains a language that is not in any level of the low hierarchy in NP. Formally, Sheu and Long [SL96] showed that $(\exists \mathcal{A})(\forall k \geq 1)\left[\Sigma_{k}^{p, \mathrm{UP}^{\mathcal{A}}} \nsubseteq \Sigma_{k}^{p, \mathcal{A}}\right]$. In their proof, they introduced special kinds of random restrictions that were motivated by, but different from, the restrictions used by Håstad [Hås87]. Using the random restrictions of Sheu and Long [SL96], we construct a relativized world $\mathcal{A}$ in which the unambiguity based hierarchies - AUPH, UPH, and $\mathcal{U P H}$ - are infinite. This extends the separation of relativized polynomial hierarchy [Yao85,Hås87] to the separations of unambiguity based relativized hierarchies. We use the same restrictions to construct an oracle $\mathcal{A}$ relative to which UAP is not contained in the polynomial hierarchy. Our separation results imply that proving that any of the unambiguity based hierarchies extend up to a finite level or proving that UAP is contained in the polynomial hierarchy is beyond the limits of relativizable proof techniques.

We now introduce certain notions that are prevalent in the theory of circuit lower bounds. We represent the variables of a circuit by $v_{z}$, for some $z \in \Sigma^{*}$. The dual of a circuit $C$ is obtained from $C$ by replacing OR gates with ANDs, AND gates with ORs, variables $x_{i}$ with $\overline{x_{i}}$, and variables $\overline{x_{j}}$ with $x_{j}$. A restriction $\rho$ of a circuit $C$ is a mapping from the variables of $C$ to $\{0,1, \star\}$. We say that a restriction $\rho$ of a circuit $C$ is a full restriction if $\rho$ assigns 0 or 1 to all the variables in $C$. Given a circuit $C$ and a restriction $\rho, C\left\lceil_{\rho}\right.$ denotes the circuit obtained from $C$ by substituting each variable $x$ with $\rho(x)$ if $\rho(x) \neq \star$. For every $A \subseteq \Sigma^{*}$, the restriction $\rho_{A}$ on the variables $v_{z}$ of a circuit $C$ is $\rho_{A}\left(v_{z}\right)=1$ if $z \in A$, and $\rho_{A}\left(v_{z}\right)=0$ if $z \notin A$. The composition of two restrictions $\rho_{1}$ and $\rho_{2}$, denoted by $\rho_{1} \rho_{2}$, is defined as follows: For every $x \in \Sigma^{*}$, $\rho_{1} \rho_{2}(x)=\rho_{2}\left(\rho_{1}(x)\right)$. 
We define specialized circuits, $\Sigma_{k}(m)$-circuits and $\Pi_{k}(m)$-circuits, used for constructing relativized worlds involving $\Sigma_{k}$ and $\Pi_{k}$ classes.

Definition 3.1 For every $m \geq 1$ and $k \geq 1, a \Sigma_{k}(m)$-circuit is a depth $k+1$ circuit with alternating $\mathrm{OR}$ and $\mathrm{AND}$ gates such that

1. the top gate, i.e., the gate at level 1 , is an $\mathrm{OR}$ gate,

2. the number of gates at level 1 to level $k-1$ is bounded by $2^{m}$,

3. the fanin of gates at level $k+1$ is $\leq m$.

$A \Pi_{k}(m)$-circuit is the dual circuit of $a \Sigma_{k}(m)$-circuit.

For every $k \geq 1$, we say that $\sigma$ is a $\Sigma_{k}^{p,(.)}$-predicate if there exist a predicate $R\left(A ; x, y_{1}, \ldots, y_{k}\right)$ over a set variable $A$ and string variables $x, y_{1}, y_{2}, \ldots, y_{k}$, and a polynomial $q$ such that the following hold: (i) $R\left(A ; x, y_{1}, y_{2}, \ldots, y_{k}\right)$ is computable in polynomial time by a deterministic oracle Turing machine that uses $A$ as the oracle and $\left\langle x, y_{1}, \ldots, y_{k}\right\rangle$ as the input and (ii) for every set $A$ and string $x, \sigma(A ; x)$ is true if and only if $\left(\exists^{q} y_{1}\right)\left(\forall^{q} y_{2}\right) \ldots\left(Q_{k}^{q} y_{k}\right) R\left(A ; x, y_{1}, y_{2}, \ldots, y_{k}\right)$ is true, where $Q_{k}=\exists$ if $k$ is odd and $Q_{k}=\forall$ if $k$ is even. We say that $\sigma$ is a $\Pi_{k}^{p,(.)}$-predicate, for $k \geq 1$, if $\neg \sigma$ is a $\Sigma_{k}^{p,(.)}$-predicate.

The following proposition states the relationship between $\Sigma_{k}^{p,(.)}$-predicates $\left(\Pi_{k}^{p,(.)}\right.$ predicates) and $\Sigma_{k}(m)$-circuits (respectively, $\Pi_{k}(m)$-circuits).

Proposition 3.2 (see [Ko89,SL94,SL96]) Let $k \geq 1$. For every $\Sigma_{k}^{p,(.)}$-predicate $\left(\Pi_{k}^{p,(.)}\right.$-predicate) $\sigma$, there is a polynomial $q($.$) such that, for all x \in \Sigma^{*}$, there is a $\Sigma_{k}(q(|x|))$-circuit (respectively, $\Pi_{k}(q(|x|))$-circuit) $C_{\sigma, x}$ with the following properties:

1. For every $A \subseteq \Sigma^{*}, C_{\sigma, x} \Gamma_{\rho_{A}}=1$ if and only if $\sigma(A ; x)$ is true, and

2. if $v_{z}$ represents a variable in $C_{\sigma, x}$, then $|z| \leq q(|x|)$.

Let $\mathcal{B}=\left\{B_{i}\right\}_{i=1}^{r}$, where $B_{i}$ 's are disjoint sets that cover the variables of $C$, and let $q$ be a real number between 0 and 1 . Sheu and Long [SL96] defined two probability spaces of restrictions, $\hat{R}_{q, \mathcal{B}}^{+}$and $\hat{R}_{q, \mathcal{B}}^{-}$, and a function $g^{\prime}$ that maps a random restriction to a restriction. A random restriction $\rho \in \hat{R}_{q, \mathcal{B}}^{+}\left(\rho \in \hat{R}_{q, \mathcal{B}}^{-}\right)$is defined as follows: For every $1 \leq i \leq r$ and for every variable $x \in B_{i}$, let $\rho(x)=\star$ with probability $q$ and $\rho(x)=1$ (respectively, $\rho(x)=0$ ) with probability $1-q$. We now define the function $g^{\prime}$ for $\rho \in \hat{R}_{q, \mathcal{B}}^{+}$. For every $1 \leq i \leq r$, let $s_{i}=\star$ with probability $q$ and let $s_{i}=0$ with probability $1-q$. Let $V_{i} \subseteq B_{i}$ be the set of variables $x$ such that $\rho(x)=\star . g^{\prime}(\rho)$ selects the variable $v$ with the highest index in $V_{i}$, assigns value $s_{i}$ to $v$, and assigns value 1 to all other variables in $V_{i}$. The function $g^{\prime}(\rho)$ for $\rho \in \hat{R}_{q, \mathcal{B}}^{-}$is defined in an analogous way by replacing 0 with 1 and vice versa.

Lemma 3.3 (Switching Lemma [SL96]) Let $C$ be a circuit consisting of an AND of ORs with bottom fanin $\leq t$. Let $\mathcal{B}=\left\{B_{i}\right\}_{i=1}^{r}$ be disjoint sets that cover the variables of $C$, and let $q$ be a real number between 0 and 1 . Then, for a random restriction $\rho \in \hat{R}_{q, \mathcal{B}}^{+}$, $\operatorname{Prob}\left[C \Gamma_{\rho g^{\prime}(\rho)}\right.$ is not equivalent to an OR of AND $s$ with bottom fanin $\left.\leq s\right] \leq \alpha^{s}$, where $\alpha<6 q t$. The above probability holds even when $\hat{R}_{q, \mathcal{B}}^{+}$is replaced by $\hat{R}_{q, \mathcal{B}}^{-}$, or when $C$ is an OR of AND $s$ and is being converted to an AND of ORs. 
Sheu and Long [SL96] defined a kind of restriction, called $U$ condition, on the assignment of variables in certain circuits. A restriction $\rho$ is said to satisfy the $\mathrm{U}$ condition if the following holds: At most one variable is assigned $\star$ or 0 in each set $B_{i}$ if $\rho$ is a random restriction from $\hat{R}_{q, \mathcal{B}}^{+}$, and at most one variable is assigned $\star$ or 1 in each set $B_{i}$ if $\rho$ is a random restriction from $\hat{R}_{q, \mathcal{B}}^{-}$[SL96]. Below, we define a global uniqueness condition (also called $G U$ condition) on full restrictions of any circuit $C$.

Definition 3.4 We say that a full restriction $\rho$ satisfies the GU condition for a circuit $C$, if the assignment of variables by $\rho$ leads to the following characteristics in the computation of $C$ :

1. If an OR gate $G_{i}$ in $C$ outputs 1 , then there is exactly one input gate to $G_{i}$ that outputs 1 , and

2. if an AND gate $G_{i}$ in $C$ outputs 0 , then there is exactly one input gate to $G_{i}$ that outputs 0 .

Theorem $3.5(\exists \mathcal{A})(\forall k \geq 1)\left[\mathrm{AU} \Sigma_{k}^{p, \mathcal{A}} \nsubseteq \Pi_{k}^{p, \mathcal{A}}\right]$.

Proof Our proof is inspired from that of Theorem 4.2 (relative to some oracle $\mathcal{D}$, for all $k \geq 1, \Sigma_{k}^{p, \text { UP }}{ }^{\mathcal{D}} \nsubseteq \Sigma_{k}^{p, \mathcal{D}}$ ) by Sheu and Long [SL96]. For every $k \geq 1$, we define a test language $L_{k}(B)$ as follows: $L_{k}(B) \subseteq 0^{*}$ such that, for every $n \in \mathbb{N}^{+}$,

$$
\begin{aligned}
& 0^{n} \in L_{k}(B) \quad \Longrightarrow\left(\exists^{n} ! y_{1}\right)\left(\forall^{n} ! y_{2}\right) \ldots\left(Q^{n} ! y_{k}\right)\left[0^{k} 1 y_{1} y_{2} \ldots y_{k} \in B\right], \text { and } \\
& 0^{n} \notin L_{k}(B) \quad \Longrightarrow\left(\forall^{n} ! y_{1}\right)\left(\exists^{n} ! y_{2}\right) \ldots\left(\bar{Q}^{n} ! y_{k}\right)\left[0^{k} 1 y_{1} y_{2} \ldots y_{k} \notin B\right],
\end{aligned}
$$

where $Q=\exists$ and $\bar{Q}=\forall$ if $k$ is odd, and $Q=\forall$ and $\bar{Q}=\exists$ if $k$ is even. Choose $\mathcal{O} \subseteq \Sigma^{*}$ such that, for every $k \geq 1, L_{k}(\mathcal{O})=0^{*}$. For every $k \geq 1$, let $\sigma_{k, 1}, \sigma_{k, 2}, \ldots$ be an enumeration of $\Sigma_{k}^{p,(.)}$-predicates. In stage $\langle k, i\rangle$, we diagonalize against $\sigma_{k, i}$ and change $\mathcal{O}$ at a certain length. Finally, let $\mathcal{A}:=\lim _{n \rightarrow \infty} \cup_{n \in \mathbb{N}^{+}} \mathcal{O}^{=n}$. We now define the stages involved in the construction of the oracle.

Stage $\langle k, i\rangle: \quad$ Choose a very large integer $n$ so that the construction in this stage does not spoil the constructions in previous stages. Also, $n$ must be large enough to meet the requirements in the proof of Claim 1. Set $\mathcal{O}:=\mathcal{O}-\Sigma^{k(n+1)+1}$. Choose a set $B \subseteq 0^{k} 1 \Sigma^{k n}$ such that the following requirement is satisfied:

$$
0^{n} \in L_{k}(B) \Longleftrightarrow \sigma_{k, i}\left(\mathcal{O} \cup B ; 0^{n}\right) \text { is true. }
$$

In Claim 1, we show that there is always a set $B \subseteq 0^{k} 1 \Sigma^{k n}$ satisfying Eqn. (3.a). Let $\mathcal{O}:=\mathcal{O} \cup B$ and move to the next stage.

\section{End of Stage}

Clearly, the existence of a set $B$ satisfying Eqn. (3.a) suffices to successfully finish stage $\langle k, i\rangle$. We now prove the statement in Claim 1.

Claim 1 In every stage $\langle k, i\rangle$, there is a set $B \subseteq 0^{k} 1 \Sigma^{k n}$ satisfying Eqn. (3.a).

Proof Assume to the contrary that in some stage $\langle k, i\rangle$, Eqn. (3.a) is not satisfied. Then, the following holds: For every $B \subseteq 0^{k} 1 \Sigma^{k n}, 0^{n} \in L_{k}(B)$ if and only if $\neg \sigma_{k, i}(\mathcal{O} \cup$ 
$\left.B ; 0^{n}\right)$ is true. We define a $C(n, k)$ circuit as follows: The depth of $C(n, k)$ is $k$, the top gate of $C(n, k)$ is an OR gate, the fanin of all the gates at level 1 to $k$ is $2^{n}$, and every leaf of $C(n, k)$ is a positive variable represented by $v_{z}$, where $z \in 0^{k} 1 \Sigma^{k n}$. The following proposition is evident.

Proposition 3.6 For every $B \subseteq 0^{k} 1 \Sigma^{k n}$,

$$
\begin{aligned}
& 0^{n} \in L_{k}(B) \Longleftrightarrow\left[\rho_{B} \text { satisfies the } \mathrm{GU} \text { condition for } C(n, k) \text { and } C(n, k) \Gamma_{\rho_{B}}=1\right], \\
& \quad \text { and } \\
& 0^{n} \notin L_{k}(B) \Longleftrightarrow\left[\rho_{B} \text { satisfies the } \mathrm{GU} \text { condition for } C(n, k) \text { and } C(n, k)\left\lceil\rho_{B}=0\right]\right. \text {. }
\end{aligned}
$$

For every $h \geq 1$, we define a family of circuits $\mathcal{F}_{k}^{h}$. Ko [Ko89] defined a $C_{k}^{h}$ circuit to be a depth $k$ circuit in $\mathcal{F}_{k}^{h}$ with fanin of gates at level $k$ exactly equal to $\sqrt{h}$ and used these circuits to separate the relativized polynomial hierarchy.

Family $\mathcal{F}_{k}^{h}$ of circuits, where $h \geq 1$ : A circuit $C$ of depth $\ell$, where $1 \leq \ell \leq k$, is in $\mathcal{F}_{k}^{h}$ if and only if the following holds:

1. $C$ has alternating OR and AND gates, and the top gate, i.e., the gate at level 1, of $C$ is an OR gate,

2. the fanin of gates at level 1 to $\ell-1$ is $h$,

3. the fanin of gates at level $\ell$ is $\geq \sqrt{h}$,

4. every leaf of $C$ is a unique positive variable.

Let $C_{\sigma_{k, i}}$ be the $\Pi_{k}\left(p_{i}(n)\right)$-circuit corresponding to $\neg \sigma_{k, i}\left((.) ; 0^{n}\right)$, for some polynomial $p_{i}$ (.). From Proposition 3.6, we wish to find a set $B \subseteq 0^{k} 1 \Sigma^{k n}$ such that (i) if $\rho_{B}$ satisfies the $G U$ condition for $C(n, k)$ and $C(n, k) \Gamma_{\rho_{B}}=1$, then $C_{\sigma_{k, i}} \Gamma_{\rho_{\mathcal{O} \cup B}}=0$, and (ii) if $\rho_{B}$ satisfies the $G U$ condition for $C(n, k)$ and $C(n, k) \Gamma_{\rho_{B}}=0$, then $C_{\sigma_{k, i}} \Gamma_{\rho_{\mathcal{O} \cup B}}=1$. Clearly, the existence of a set $B$ satisfying (i) and (ii) suffices to prove the claim. Next, we describe our approach to show the existence of such a set $B$.

We define a restriction $\hat{\rho}_{\mathcal{O}}$ on $C_{\sigma_{k, i}}$ as follows: For every variable $v_{z}$ in $C_{\sigma_{k, i}}$, if $z \in \mathcal{O}$ then let $\hat{\rho}_{\mathcal{O}}\left(v_{z}\right)=1$, if $z \notin \mathcal{O} \cup 0^{k} 1 \Sigma^{k n}$ then let $\hat{\rho}_{\mathcal{O}}\left(v_{z}\right)=0$, and if $z \in 0^{k} 1 \Sigma^{k n}$ then let $\hat{\rho}_{\mathcal{O}}\left(v_{z}\right)=\star$. Let $C_{\sigma_{k, i}(\mathcal{O})}={ }_{d f} C_{\sigma_{k, i}} \Gamma_{\hat{\rho}_{\mathcal{O}}}$. Thus, the only variables $v_{z}$ appearing in $C_{\sigma_{k, i}(\mathcal{O})}$ are the ones for which $z \in 0^{k} 1 \Sigma^{k n}$. Suppose that no set $B \subseteq 0^{k} 1 \Sigma^{k n}$ satisfying (i) and (ii) exists. Then, the following holds: For every $B \subseteq 0^{k} 1 \Sigma^{k n}$,

$\rho_{B}$ satisfies the $G U$ condition for $C(n, k)$ and $C(n, k)\left\lceil_{\rho_{B}}=C_{\sigma_{k, i}(\mathcal{O})}\left\lceil_{\rho_{B}}=1\right.\right.$,

or

$\rho_{B}$ satisfies the $G U$ condition for $C(n, k)$ and $C(n, k)\left\lceil_{\rho_{B}}=C_{\sigma_{k, i}(\mathcal{O})}\left\lceil_{\rho_{B}}=0\right.\right.$.

Lemma 3.7 For every $1 \leq \ell \leq k$ and for all sufficiently large $h$, for any circuit $C_{\mathcal{F}} \in \mathcal{F}_{k}^{h}$ of depth $\ell$, and for any $\Pi_{\ell}(m)$-circuit $C_{\pi}$, if it holds that

(for every full restriction $\rho$ satisfying the GU condition for $C_{\mathcal{F}}$ ) $\left[C_{\mathcal{F}} \Gamma_{\rho}=C_{\pi}\left\lceil_{\rho}\right]\right.$,

then $m \geq \delta \cdot h^{1 / 3}$, where $\delta=1 / 12$.

Proof The proof is similar to that of Theorem 4.1 by Sheu and Long [SL96]. We prove the lemma by induction on $\ell$. For the base case, i.e., $\ell=1$, let $C_{\mathcal{F}}$ be an arbitrary 
OR gate with $\geq \sqrt{h}$ variables. Let $C_{\pi}$ be an arbitrary $\Pi_{1}(t)$-circuit, where $t<\delta \cdot h^{1 / 3}$. Note that $C_{\pi}$ is an AND of ORs with bottom fanin $\leq t$. We will show that there is a full restriction $\rho$ satisfying the $G U$ condition for $C_{\mathcal{F}}$ such that $C_{\mathcal{F}}\left\lceil\rho \neq C_{\pi}\lceil\rho\right.$. This will give a contradiction, and so it must be that $\lambda_{1}(h) \geq \delta \cdot h^{1 / 3}$. We consider the following cases.

Case $C_{\pi} \Gamma_{\rho_{\emptyset}}=0$ : Then, there is an OR gate $G_{i}$ in $C_{\pi}$ such that all the variables in $G_{i}$ are positive, i.e., $G_{i} \Gamma_{\rho_{\emptyset}}=0$. Since $t<\delta \cdot h^{1 / 3}<\sqrt{h}$ for sufficiently large $h$, there is a variable $v_{z}$ in $C_{\mathcal{F}}$ but not occurring in $G_{i}$. Then, $\rho_{\{z\}}$ satisfies the $G U$ condition for $C_{\mathcal{F}}, C_{\mathcal{F}} \Gamma_{\rho_{\{z\}}}=1$, and $C_{\pi} \Gamma_{\rho_{\{z\}}}=0$.

Case $C_{\pi} \Gamma_{\rho_{\emptyset}}=1$ : This immediately yields a contradiction, since $\rho_{\emptyset}$ satisfies the $G U$ condition for $C_{\mathcal{F}}, C_{\mathcal{F}} \Gamma_{\rho_{\emptyset}}=0$, and $C_{\pi} \Gamma_{\rho_{\emptyset}}=1$.

Induction Hypothesis: For every depth $\ell$ circuit $C_{\mathcal{F}} \in \mathcal{F}_{k}^{h}$ and for every $\Pi_{\ell}(t)$ circuit $C_{\pi}$, where $t<\delta \cdot h^{1 / 3}$, there is a full restriction $\varpi$ satisfying the $G U$ condition for $C_{\mathcal{F}}$ such that $C_{\mathcal{F}}\left\lceil_{\rho} \neq C_{\pi} \Gamma_{\rho}\right.$.

Induction Step: Let $C_{\mathcal{F}}$ be an arbitrary depth $\ell+1$ circuit in $\mathcal{F}_{k}^{h}$ and let $C_{\pi}$ be an arbitrary $\Pi_{\ell+1}(t)$-circuit, where $t<\delta \cdot h^{1 / 3}$. Let $G_{1}, G_{2}, \ldots, G_{r}$ be the bottom OR gates of $C_{\mathcal{F}}$ if $\ell+1$ is odd, or the bottom AND gates of $C_{\mathcal{F}}$ if $\ell+1$ is even. Let $q=h^{-1 / 3}$ and let $\mathcal{B}=\left\{B_{i}\right\}_{i=1}^{r}$, where $B_{i}$ is the set of variables in $G_{i}$. We first show that, for a randomly chosen restriction $\rho$, where $\rho \in \hat{R}_{q, \mathcal{B}}^{+}$if $\ell+1$ is even and $\rho \in \hat{R}_{q, \mathcal{B}}^{-}$if $\ell+1$ is odd, the following is true.

(a) With probability $\geq 3 / 4, C_{\mathcal{F}} \Gamma_{\rho g^{\prime}(\rho)}$ is a depth $\ell$ circuit in $\mathcal{F}_{k}^{h}$, and

(b) with probability $\geq 3 / 4, C_{\pi}\left\lceil_{\rho g^{\prime}(\rho)}\right.$ is equivalent to a $\Pi_{\ell}(t)$-circuit.

Lemma 3.8 shows that (a) is true and Lemma 3.9 shows that (b) is true. It follows that there is a restriction $\rho$ such that $C_{\mathcal{F}} \Gamma_{\rho g^{\prime}(\rho)}$ is a depth $\ell$ circuit in $\mathcal{F}_{k}^{h}$ and $C_{\pi} \Gamma_{\rho g^{\prime}}(\rho)$ is equivalent to a $\Pi_{\ell}(t)$-circuit. By induction hypothesis, there is a full restriction $\varpi$ satisfying the $G U$ condition for $C_{\mathcal{F} \Gamma_{\rho g^{\prime}(\rho)}}$ such that $C_{\mathcal{F}} \Gamma_{\rho g^{\prime}(\rho) \varpi} \neq C_{\pi} \Gamma_{\rho g^{\prime}(\rho) \varpi}$. Now it only remains to show that $\rho g^{\prime}(\rho) \varpi$ satisfies the $G U$ condition for $C_{\mathcal{F}}$.

The probability spaces, $\hat{R}_{q, \mathcal{B}}^{+}$and $\hat{R}_{q, \mathcal{B}}^{-}$, of restrictions, and function $g^{\prime}$ are defined in such a way that if the bottom level gates $G_{i}$ 's in $C_{\mathcal{F}}$ are ANDs, then the restriction $\rho g^{\prime}(\rho)$ assigns at most one variable to 0 or $\star$ in each $G_{i}$, and if the bottom level gates $G_{j}$ 's in $C_{\mathcal{F}}$ are ORs, then the restriction $\rho g^{\prime}(\rho)$ assigns at most one variable to 1 or $\star$ in each $G_{j}$. Thus, the restriction $\rho g^{\prime}(\rho)$ enforces unambiguity at bottom level gates of $C_{\mathcal{F}}$. Since $\varpi$ is a full restriction satisfying the $G U$ condition for $C_{\mathcal{F}} \Gamma_{\rho g^{\prime}}(\rho), \rho g^{\prime}(\rho) \varpi$ enforces unambiguity in $C_{\mathcal{F}}$. It follows that $\rho g^{\prime}(\rho) \varpi$ satisfies the $G U$ condition for $C_{\mathcal{F}}$.

(Lemma 3.7)

Since $C(n, k) \in \mathcal{F}_{k}^{2^{n}}, C_{\sigma_{k, i}(\mathcal{O})}$ is a $\Pi_{k}\left(p_{i}(n)\right)$ circuit, and $p_{i}(n)=o\left(2^{n / 3}\right)$, we get a contradiction with Eqn. (3.b) and Lemma 3.7.

(Claim 1 and Theorem 3.5)

Lemma 3.8 Let $k \geq 2$, let $1 \leq \ell<k$ and let $h$ be any sufficiently large integer. Let $C_{\mathcal{F}}$ be a depth $\ell+1$ circuit in $\mathcal{F}_{k}^{h}$. Let $G_{1}, G_{2}, \ldots, G_{r}$ be the bottom level gates of $C_{\mathcal{F}}$, let $q=h^{-1 / 3}$, and let $\mathcal{B}=\left\{B_{i}\right\}_{i=1}^{r}$, where $B_{i}$ is the set of variables in $G_{i}$. Then, for a 
random restriction $\rho$, where $\rho \in \hat{R}_{q, \mathcal{B}}^{+}$if $\ell+1$ is even, and $\rho \in \hat{R}_{q, \mathcal{B}}^{-}$if $\ell+1$ is odd, the following holds:

$$
\operatorname{Prob}\left[C_{\mathcal{F}} \Gamma_{\rho g^{\prime}(\rho)} \text { is a depth } \ell \text { circuit in } \mathcal{F}_{k}^{h}\right] \geq \frac{3}{4} .
$$

Proof The proof is similar to that of Lemma 6.8 by Håstad [Hås87]. Let the bottom level gates $G_{1}, G_{2}, \ldots, G_{r}$ of $C_{\mathcal{F}}$ be AND gates. (The case when the bottom level gates of $C_{\mathcal{F}}$ are ORs is similar.) Then, for a random restriction $\rho \in \hat{R}_{q, \mathcal{B}}^{+}, C_{\mathcal{F}} \Gamma_{\rho g^{\prime}(\rho)} \notin \mathcal{F}_{k}^{h}$ if and only if there is an OR gate at level $\ell$ in $C_{\mathcal{F}} \Gamma_{\rho g^{\prime}(\rho)}$ with bottom fanin $<\sqrt{h}$. Let $p_{i}=_{d f}\left(\begin{array}{c}h \\ i\end{array}\right) q^{\prime i}\left(1-q^{\prime}\right)^{h-i}$ denote the probability that an OR gate at level $\ell$ in $C_{\mathcal{F}} \Gamma_{\rho g^{\prime}(\rho)}$ has bottom fanin exactly equal to $i$, where $q^{\prime}$ is the probability that the restriction $\rho g^{\prime}(\rho)$ assigns an input of an OR gate at level $\ell$ to $\star$. (Note that $q^{\prime} \geq q\left(1-e^{-q \sqrt{h}}\right)$.) Then,

$$
\operatorname{Prob}_{\rho \in \hat{R}_{q, \mathcal{B}}^{+}}\left[C_{\mathcal{F}}\left\lceil_{\rho g^{\prime}(\rho)} \notin \mathcal{F}_{k}^{h}\right] \leq h^{\ell-1} \times\left[\sum_{i=0}^{\sqrt{h}-1} p_{i}\right] .\right.
$$

We now obtain an upper bound on the r.h.s. of Eqn. (3.c). It can be easily verified that, for all sufficiently large $h$ and for every $1 \leq i \leq \sqrt{2 h}-1, \frac{p_{i}}{p_{i-1}} \geq 2$. Then,

$$
\sum_{i=0}^{\sqrt{h}-1} p_{i} \leq p_{\sqrt{h}-1} \times\left(\sum_{i=0}^{\infty} 2^{-i}\right) \leq 2 \cdot p_{\sqrt{h}-1} .
$$

Also, $p_{\sqrt{h}-1} \leq 2^{-(\sqrt{2 h}-\sqrt{h})} \cdot p_{\sqrt{2 h}-1} \leq 2^{-\sqrt{h} / 3}$, since $p_{\sqrt{2 h}-1} \leq 1$. Thus, it follows that

$$
\operatorname{Prob}_{\rho \in \hat{R}_{q, \mathcal{B}}^{+}}\left[C_{\mathcal{F}}\left\lceil_{\rho g^{\prime}(\rho)} \notin \mathcal{F}_{k}^{h}\right] \leq \frac{2 \cdot h^{\ell-1}}{2^{\sqrt{h} / 3}} \leq \frac{1}{4},\right.
$$

for sufficiently large $h$.

(Lemma 3.8)

Lemma 3.9 is a restatement of Lemma 3.6 by Sheu and Long [SL96] with different parameter values. So, we omit the proof of Lemma 3.9 .

Lemma 3.9 Let $k \geq 2$, let $1 \leq \ell<k$, let $\delta=1 / 12$, and let $t$ be an integer. Let $C_{\pi}$ be an arbitrary $\Sigma_{\ell+1}(t)$-circuit $\left(\Pi_{\ell+1}(t)\right.$-circuit). Let $V$ be the set of variables in $C_{\pi}$, let $q=\delta / t$, and let $\mathcal{B}=\left\{B_{i}\right\}_{i=1}^{r}$ be an arbitrary partition of the variables in $V$. Then, for a random restriction $\rho$, where $\rho \in \hat{R}_{q, \mathcal{B}}^{+}$or $\rho \in \hat{R}_{q, \mathcal{B}}^{-}$, the following holds:

$\operatorname{Prob}\left[C_{\pi} \Gamma_{\rho g^{\prime}(\rho)}\right.$ is equivalent to a $\Sigma_{\ell}(t)$-circuit (respectively, $\Pi_{\ell}(t)$-circuit) $] \geq \frac{3}{4}$.

Proof Omitted (see Lemma 3.6 of [SL96]).

The following corollary is an easy consequence of Theorem 3.5.

Corollary 3.10 There is an oracle $\mathcal{A}$ relative to which the alternating unambiguous polynomial hierarchy AUPH, the unambiguous polynomial hierarchy UPH, the promise unambiguous polynomial hierarchy $\mathcal{U P H}$, and the polynomial hierarchy $\mathrm{PH}$ are infinite. 
Note that Theorem 3.5 does not imply relativized separation of UAP from $\mathrm{PH}$ in any obvious way. Theorem 3.11 achieves this separation using the proof techniques of Theorem 3.5.

Theorem $3.11(\exists \mathcal{A})\left[\mathrm{UAP}^{\mathcal{A}} \nsubseteq \mathrm{PH}^{\mathcal{A}}\right]$.

Proof The proof is almost the same as that of Theorem 3.5. We construct an oracle $\mathcal{A}$ and a test language $L(\mathcal{A}) \in \mathrm{UAP}^{\mathcal{A}}$ such that, for every $k \geq 1, L(\mathcal{A}) \notin \Pi_{k}^{p, \mathcal{A}}$. Clearly, this suffices to prove the theorem. For every $B \subseteq \Sigma^{*}$, our test language $L(B)$ is defined as follows: $L(B) \subseteq 0^{*}$ such that, for every $n \in \mathbb{N}^{+}$,

$$
\begin{aligned}
& 0^{n} \in L(B) \quad \Longrightarrow\left(\exists^{n} ! y_{1}\right)\left(\forall^{n} ! y_{2}\right) \ldots\left(Q_{n}^{n} ! y_{n}\right)\left[y_{1} y_{2} \ldots y_{n} \in B\right], \text { and } \\
& 0^{n} \notin L(B) \quad \Longrightarrow\left(\forall^{n} ! y_{1}\right)\left(\exists^{n} ! y_{2}\right) \ldots\left(\bar{Q}_{n}^{n} ! y_{n}\right)\left[y_{1} y_{2} \ldots y_{n} \notin B\right],
\end{aligned}
$$

where $Q_{n}=\exists$ and $\bar{Q}_{n}=\forall$ if $n$ is odd, and $Q_{n}=\forall$ and $\bar{Q}_{n}=\exists$ if $n$ is even. Choose $\mathcal{O} \subseteq \Sigma^{*}$ such that $L(\mathcal{O})=0^{*}$. For every $k \geq 1$, let $\sigma_{k, 1}, \sigma_{k, 2}, \ldots$ denote an enumeration of $\Sigma_{k}^{p,(.)}$-predicates. In stage $\langle k, i\rangle$, we diagonalize against $\sigma_{k, i}$ and change $\mathcal{O}$ at a certain length. Finally, let $\mathcal{A}:=\lim _{n \rightarrow \infty} \cup_{n \in \mathbb{N}^{+}} \mathcal{O}^{=n}$.

Stage $\langle k, i\rangle$ : Choose a very large integer $n$ so that the construction in this stage does not affect the constructions in previous stages and the requirements in the proof of Claim 2 are met. Set $\mathcal{O}:=\mathcal{O}-\Sigma^{n^{2}}$. Choose a set $B \subseteq \Sigma^{n^{2}}$ such that the following requirement is satisfied:

$$
0^{n} \in L(B) \Longleftrightarrow \sigma_{k, i}\left(\mathcal{O} \cup B ; 0^{n}\right) \text { is true. }
$$

Claim 2 shows that there is always a set $B \subseteq \Sigma^{n^{2}}$ satisfying Eqn. (3.d). Let $\mathcal{O}:=\mathcal{O} \cup B$ and move to the next stage.

\section{End of Stage}

Claim 2 In every stage $\langle k, i\rangle$, there is a set $B \subseteq \Sigma^{n^{2}}$ satisfying Eqn. (3.d).

Proof Assume to the contrary that in some stage $\langle k, i\rangle$, Eqn. (3.d) is not satisfied. Let $C(n)$ denote the following circuit: The depth of $C(n)$ is $n$, the top gate of $C(n)$ is an OR gate, the fanin of all the gates at level 1 to $n$ is $2^{n}$, and every leaf of $C(n)$ is a positive variable represented by $v_{z}$, where $z \in \Sigma^{n^{2}}$. We define, for every $h \geq 1$, a family $\mathcal{F}_{n}^{h}$ of circuits in a similar way as $\mathcal{F}_{k}^{h}$ is defined in the proof of Claim 1, except that the variable $k$ is replaced by $n$ throughout the definition of $\mathcal{F}_{k}^{h}$. Let $C_{\sigma_{k, i}}$ be the $\Pi_{k}\left(p_{i}(n)\right)$-circuit corresponding to $\neg \sigma_{k, i}\left((.) ; 0^{n}\right)$.

Next, we define a restriction $\hat{\rho}_{\mathcal{O}}$ as follows: For every variable $v_{z}$ in $C_{\sigma_{k, i}}$, if $z \in \mathcal{O}$ then $\hat{\rho}_{\mathcal{O}}\left(v_{z}\right)=1$, if $z \notin \mathcal{O} \cup \Sigma^{n^{2}}$ then $\hat{\rho}_{\mathcal{O}}\left(v_{z}\right)=0$, and if $z \in \Sigma^{n^{2}}$ then $\hat{\rho}_{\mathcal{O}}\left(v_{z}\right)=\star$. Let $C_{\sigma_{k, i}(\mathcal{O})}={ }_{d f} C_{\sigma_{k, i}} \Gamma_{\hat{\rho} \mathcal{O}}$. The following equation follows from our assumptions: For every $B \subseteq \Sigma^{n^{2}}$,

$$
\begin{aligned}
& \rho_{B} \text { satisfies the } G U \text { condition for } C(n) \text { and } C(n)\left\lceil\rho_{B}=C_{\sigma_{k, i}(\mathcal{O})}\left\lceil\rho_{B}=1,\right.\right. \\
& \qquad o r \\
& \rho_{B} \text { satisfies the } G U \text { condition for } C(n) \text { and } C(n)\left\lceil\rho_{B}=C_{\sigma_{k, i}(\mathcal{O})}\left\lceil\rho_{B}=0 .\right.\right.
\end{aligned}
$$

The following lemma is a simple extension of Lemma 3.7. 
Lemma 3.12 For every $1 \leq \ell^{\prime} \leq n$ and $1 \leq \ell \leq \min \left\{\ell^{\prime}, k\right\}$, for all sufficiently large $h$, for any circuit $C_{\mathcal{F}} \in \mathcal{F}_{n}^{h}$ of depth $\ell^{\prime}$, and for any $\Pi_{\ell}(m)$-circuit $C_{\pi}$, if it holds that,

(for every full restriction $\rho$ satisfying the GU condition for $C_{\mathcal{F}}$ ) $\left[C_{\mathcal{F}}\left\lceil_{\rho}=C_{\pi}\left\lceil_{\rho}\right]\right.\right.$,

then $m=\Omega\left(h^{1 / 3}\right)$.

Proof Omitted, since the proof is similar to that of Lemma 3.7.

Since $C(n) \in \mathcal{F}_{n}^{2^{n}}, C_{\sigma_{k, i}(\mathcal{O})}$ is a $\Pi_{k}\left(p_{i}(n)\right)$-circuit, and $p_{i}(n)=o\left(2^{n / 3}\right)$, we get a contradiction with Eqn. (3.e) and Lemma 3.12. (Claim 2 and Theorem 3.11)

Crâsmaru et al. [CGRS04] showed that there is an oracle relative to which UAP $\neq$ $\mathcal{U} \Sigma_{2}^{p}$. Corollary 3.13 shows that in some relativized world, UAP is much more powerful than the promise unambiguous polynomial hierarchy $\mathcal{U P H}$. Thus, Corollary 3.13 is a strengthening of their result.

Corollary 3.13 There is an oracle relative to which $\mathcal{U P H} \subset \mathrm{UAP}$.

\section{Complexity of Unambiguous Alternating Solution}

Wagner studied the class $\nabla \mathrm{P}$, denoted by UAS in this paper, of all sets that are accepted by polynomial-time alternating Turing machines with partially defined AND and OR functions. UAS is a natural class with complete sets and is related with UAP in the same way as US [BG82] is related with UP. We define a variant of UAS, denoted by $\mathrm{UAS}(k)$, where the number of alternations allowed is bounded by some constant $k \geq 1$, instead of the unbounded number of alternations in the definition of UAS. (Thus, UAS(1) is the same as the unique solution class US.)

Definition 4.1 [Wag92] The class UAS, denoted by $\nabla \mathrm{P}$ in [Wag92], is the class of all sets $L \subseteq \Sigma^{*}$ for which there exist polynomials $p($.$) and q($.$) , and a polynomial-time$ computable predicate $R$ such that

$$
L=\left\{x \in \Sigma^{*} \mid\left(\exists^{p} ! y_{1}\right)\left(\forall^{p} ! y_{2}\right) \ldots\left(Q^{p} ! y_{q}\right) R\left(x, y_{1}, y_{2}, \ldots, y_{q}\right)\right\},
$$

where $Q=\exists$ if $q(|x|)$ is odd and $Q=\forall$ if $q(|x|)$ is even.

The class $\operatorname{UAS}(k)$, for every $k \geq 1$, consists of all sets for which strings in the set are accepted unambiguously by some polynomial-time alternating Turing machine $N$ with at most $k$ alternations, while strings not in the set either are rejected or are accepted with ambiguity by $N$. A formal definition is as follows.

Definition 4.2 The class $\mathrm{UAS}(k)$, for $k \geq 1$, is the class of all sets $L \subseteq \Sigma^{*}$ for which there exist a polynomial $p($.$) and a polynomial-time computable predicate R$ such that

$$
L=\left\{x \in \Sigma^{*} \mid\left(\exists^{p} ! y_{1}\right)\left(\forall^{p} ! y_{2}\right) \ldots\left(Q^{p} ! y_{k}\right) R\left(x, y_{1}, y_{2}, \ldots, y_{k}\right)\right\},
$$

where $Q=\exists$ if $k$ is odd and $Q=\forall$ if $k$ is even.

Theorem 4.3 1. $\mathrm{UAS} \subseteq \mathrm{C}=\mathrm{P}$ and $\mathrm{UAS} \subseteq \forall \oplus \mathrm{P}[\mathrm{Wag} 92]$.

2. For every $k \geq 1$, $\mathrm{UP} \subseteq \mathrm{US} \subseteq \mathrm{UAS}(k) \subseteq \mathrm{UAS}(k+1) \subseteq \mathrm{UAS}$. 


\section{For every $k \geq 1, \operatorname{AU} \Sigma_{k}^{p} \subseteq \mathrm{UAS}(k)$.}

Theorem 4.4 shows that relative to an oracle $\mathcal{A}$, for all $k \geq 1, \mathrm{UP}_{\leq k+1}^{\mathcal{A}}$ is not contained in $\operatorname{UAS}(k)^{\mathcal{A}}$. Thus relative to the same oracle, bounded ambiguity classes $\mathrm{UP}_{\leq k}$ and bounded-level unambiguous alternating solution classes $\mathrm{UAS}(k)$, for $k \geq 1$, form infinite hierarchies. Theorem 4.4 also implies that there is a relativized world where for all $k \geq 1$, $\mathrm{UP} \mathrm{P}_{\leq k+1}$ is not contained in $\mathrm{AU} \Sigma_{k}^{p}$. In contrast, Lange and Rossmanith [LR94] proved that FewP $\subseteq \mathcal{U} \Sigma_{2}^{p}$ in every relativized world. It follows that relative to the oracle of Theorem 4.4, for all $k \geq 1, \mathcal{U} \Sigma_{2}^{p, \mathcal{A}} \nsubseteq \mathrm{AU} \Sigma_{k}^{p, \mathcal{A}}$.

The proof of Theorem 4.4 uses Lemmas 4.6, 4.7, 4.9, and 4.10. We first give a proof of Theorem 4.4 assuming that Lemmas 4.6, 4.7, 4.9, and 4.10 hold. Later at the end of the proof of Theorem 4.4, we present the proofs of these lemmas.

Theorem $4.4(\exists \mathcal{A})(\forall k \geq 1)\left[\mathrm{UP}_{\leq k+1}^{\mathcal{A}} \nsubseteq \mathrm{UAS}^{(k)^{\mathcal{A}}}\right]$.

Proof For every $k \in \mathbb{N}^{+}$, we define our test language $L_{k}(B)$ as follows:

$$
L_{k}(B)=\left\{0^{k} 10^{n} \mid B \cap 0^{k} 1 \Sigma^{n} \neq \emptyset\right\} .
$$

We will construct an oracle $\mathcal{A}$ such that $\mathcal{A} \subseteq 0\{0\}^{*} 1\{0,1\}^{*}$ and for every $k, n \in \mathbb{N}^{+}$, $\left\|\mathcal{A} \cap 0^{k} 1 \Sigma^{n}\right\| \leq k+1$. This will guarantee that, for every $k \in \mathbb{N}^{+}, L_{k}(\mathcal{A})$ is in $\operatorname{UP}_{<k+1}^{\mathcal{A}}$. For every $k \in \mathbb{N}^{+}$, let $N_{k, 1}, N_{k, 2}, N_{k, 3}, \ldots$ be an enumeration of polynomial-time bounded normalized oracle ATMs such that, for every oracle $B$ and for every $i \in \mathbb{N}^{+}$, $N_{k, i}^{B}$ is a $k$-level ATM and $p_{i}(n)={ }_{d f} n^{i}+i$ is the switch time of $N_{k, i}^{B}$. Let $\mathcal{O}:=\emptyset$. In stage $\langle k, i\rangle$, we diagonalize against $N_{k, i}$ and change $\mathcal{O}$ at a certain length. At the end of every stage, let $\mathcal{A}:=\bigcup_{n \in \mathbb{N}^{+}} \mathcal{O}^{=n}$.

Stage $\langle k, i\rangle$ : Choose $n$ large enough such that (a) no string of length $n$ or more is queried by machines considered in previous stages and (b) $2^{\frac{n-2}{4^{k}}} \gg 64 p_{i}^{2}(n)$. If $N_{k, i}^{\mathcal{O}}\left(0^{n}\right)$ accepts with unambiguity, then move to the next stage. Otherwise, choose a set $S \subseteq 0^{k} 1 \Sigma^{n}$ such that $1 \leq\|S\| \leq k+1$ and $N_{k, i}^{\mathcal{O} \cup S}\left(0^{n}\right)$ rejects with unambiguity or loses unambiguity somewhere in its computation tree. In that case, let $\mathcal{O}:=\mathcal{O} \cup S$ and move to the next stage.

End of Stage

Henceforward, we fix a $k \in \mathbb{N}^{+}$. If, for every $i \in \mathbb{N}^{+}$, the construction in stage $\langle k, i\rangle$ is feasible, then clearly $L_{k}(\mathcal{A}) \in \mathrm{UP}_{\leq k+1}^{\mathcal{A}}$ and $L_{k}(\mathcal{A}) \notin \mathrm{UAS}(k)^{\mathcal{A}}$. The rest of the proof is devoted to showing that the construction in stage $\langle k, i\rangle$ is feasible, for every $i \in \mathbb{N}^{+}$. Suppose that for some $i \geq 1$, the construction in stage $\langle k, i\rangle$ is not feasible. Then, the following hold:

1. $N_{k, i}^{\mathcal{O}}\left(0^{n}\right)$ rejects with unambiguity or loses unambiguity somewhere in its computation tree, and

2. For every $S \subseteq 0^{k} 1 \Sigma^{n}$ such that $1 \leq\|S\| \leq k+1, N_{k, i}^{\mathcal{O} U S}\left(0^{n}\right)$ accepts with unambiguity.

If $N_{k, i}^{\mathcal{O}}\left(0^{n}\right)$ loses unambiguity, then $\operatorname{ctree}\left(N_{k, i}, \mathcal{O}, 0^{n}\right)$ lacks the nice structure which every unambiguous computation tree possesses. However, Lemma 4.7 states that there is a salient node $\vartheta$ in ctree $\left(N_{k, i}, \mathcal{O}, 0^{n}\right)$ and a set $S_{\vartheta} \subseteq 0^{k} 1 \Sigma^{n}$ satisfying the properties 1,2 , and 3 of the lemma. Instead of working with the root $\varphi$ of $\operatorname{ctree}\left(N_{k, i}, \mathcal{O}, 0^{n}\right)$ and 
the set $0^{k} 1 \Sigma^{n}$, we will now work with the node $\vartheta$ and the set $S_{\vartheta} \subseteq 0^{k} 1 \Sigma^{n}$ as promised in Lemma 4.7. We will see that the size of $S_{\vartheta}$ and the properties of $\vartheta$ mentioned in Lemma 4.7 are enough to obtain a contradiction with our initial assumption. W.l.o.g., we assume that $\vartheta$ is an existential node; in case $\vartheta$ is a universal node, then a similar argument will apply.

Note that Lemma 4.7 implies that $\left\|S_{\vartheta}\right\| \geq 2^{n-1}-\frac{k \cdot p(n)}{2}>2^{n-2}$. If $\vartheta$ is a salient node at level $k$ in $\operatorname{ctree}\left(N_{k, i}, \mathcal{O}, 0^{n}\right)$, then Lemma 4.9 implies that there exist $\alpha, \beta \in S_{\vartheta}$ such that subtree $\left(N_{k, i}, \mathcal{O} \cup\{\alpha, \beta\}, 0^{n}, \vartheta\right)$ is ambiguous. This gives a contradiction with the assumption that, for every $S \subseteq 0^{k} 1 \Sigma^{n}$ such that $1 \leq\|S\| \leq k+1, N_{k, i}^{\mathcal{O} \cup S}\left(0^{n}\right)$ is unambiguous. So, we now assume that $1 \leq \operatorname{level}(\vartheta)=r<k$. From Lemma 4.10 with parameter $\ell=0$, it follows that there is a set $T_{\eta} \subseteq S_{\vartheta}$ with $0 \leq\left\|T_{\eta}\right\| \leq 1$, a node $\eta$ in subtree $\left(N_{k, i}, \mathcal{O}, 0^{n}, \vartheta\right)$, and a set $S_{\eta} \subseteq S_{\vartheta}$ satisfying the properties $1,2,3$ and 4 of the lemma. In particular, $\left\|S_{\eta}\right\| \geq\left\|S_{\vartheta}\right\|^{1 / 4}>2^{\frac{n-2}{4}}$ and $r+1 \leq \operatorname{level}(\eta) \leq k$. Note that our initial assumption about the existence of a salient node $\vartheta$ at level $r$ is reduced to an assumption about the existence of another salient node $\eta$ at a higher level, where $r+1 \leq \operatorname{level}(\eta) \leq k$, in $\operatorname{ctree}\left(N_{k, i}, \mathcal{O}, 0^{n}\right)$. (This suggests the inductive approach of the proof.) If level $(\eta)=k$, then, as explained earlier, Lemma 4.9 implies a contradiction. Otherwise, we iteratively apply Lemma 4.10 with parameter $\ell$ varying from 1 to at most $k-(1+r)$. (We need at most $k-(1+r)$ iterations, since in each iteration we move down at least one level in ctree $\left(N_{k, i}, \mathcal{O}, 0^{n}\right)$.) After all the iterations, we will end up with a node $\zeta$ at level $k$, a set $S_{\zeta}$ with $\left\|S_{\zeta}\right\| \geq 2^{\frac{n-2}{4^{k-r}}} \geq 2^{\frac{n-2}{4^{k}}} \gg 64 p^{2}(n)$ and a set $\mathcal{O}^{\prime} \subseteq 0^{k} 1 \Sigma^{n}$ of size $\leq k-r$ such that (a) if $\zeta$ is an existential node, then subtree $\left(N_{k, i}, \mathcal{O} \cup \mathcal{O}^{\prime}, 0^{n}, \zeta\right)$ rejects and for every $\alpha \in S_{\zeta}$, subtree $\left(N_{k, i}, \mathcal{O} \cup \mathcal{O}^{\prime} \cup\{\alpha\}, 0^{n}, \zeta\right)$ accepts with unambiguity, and (b) if $\zeta$ is a universal node, then $\operatorname{subtree}\left(N_{k, i}, \mathcal{O} \cup \mathcal{O}^{\prime}, 0^{n}, \zeta\right)$ accepts, and for every $\alpha \in S_{\zeta}$, subtree $\left(N_{k, i}, \mathcal{O} \cup \mathcal{O}^{\prime} \cup\{\alpha\}, 0^{n}, \zeta\right)$ rejects with unambiguity. From Lemma 4.9, it follows that there exist $\alpha, \beta \in S_{\zeta}$ such that subtree $\left(N_{k, i}, \mathcal{O} \cup \mathcal{O}^{\prime} \cup\{\alpha, \beta\}, 0^{n}, \zeta\right)$ is ambiguous. Since $\left\|\mathcal{O}^{\prime} \cup\{\alpha, \beta\}\right\| \leq k-r+2 \leq k+1$, we get a contradiction with our initial assumption.

(Theorem 4.4)

Corollary 4.5 There is an oracle $\mathcal{A}$ such that, for every $k \geq 1, \mathrm{UP}_{\leq k}^{\mathcal{A}} \subset \mathrm{UP}_{\leq k+1}^{\mathcal{A}}$, $\operatorname{AU} \Sigma_{k}^{p, \mathcal{A}} \subset \operatorname{AU} \Sigma_{k+1}^{p, \mathcal{A}}, \operatorname{UAS}(k)^{\mathcal{A}} \subset \operatorname{UAS}(k+1)^{\mathcal{A}}$, and $\mathcal{U} \Sigma_{2}^{p, \mathcal{A}} \nsubseteq \mathrm{AU} \Sigma_{k}^{p, \mathcal{A}}$.

Lemma 4.6, called Party Lemma in $\left[\mathrm{CGH}^{+} 89\right]$, states the following combinatorial fact. Suppose that $S_{i}$ denotes the set of names of people that person $i$ knows in a party of $\ell$ people. If $\sum\left\|S_{i}\right\|$ is small, then there exists a set $T$ of perfect strangers in the sense that any person $e$ in $T$ does not know the name of any other person, other than itself, in $T$.

Lemma 4.6 (Party Lemma $\left[\mathbf{C G H}^{+} \mathbf{8 9}\right]$ ) For every $\ell, k \geq 2$ and for all nonempty sets $S_{1}, \ldots, S_{\ell} \subseteq \mathbb{N}^{+}$such that $\sum_{i=1}^{\ell}\left\|S_{i}\right\|<\frac{\ell(\ell-1)}{k(k-1)}$, there exists a set $T \subseteq\{1,2, \ldots, \ell\}$ with $\|T\|=k$ such that, for all $e \in T, S_{e} \cap(T-\{e\})=\emptyset$.

Informally, Lemma 4.7 states that if $N$ is a polynomial-time bounded normalized ATM such that, for every oracle $B \subseteq \Sigma^{*}, N^{B}$ is a $k$-level ATM with a fixed switch time, and if $\mathcal{O}$ is an arbitrary partial oracle such that $\mathcal{O} \cap 0^{k} 1 \Sigma^{n}=\emptyset$, then the following is true: If, for every $\alpha \in 0^{k} 1 \Sigma^{n}, N^{\mathcal{O} \cup\{\alpha\}}\left(0^{n}\right)$ accepts with unambiguity and $N^{\mathcal{O}}\left(0^{n}\right)$ does not accept with unambiguity, then there is a salient node $\vartheta$ in $\operatorname{ctree}\left(N, \mathcal{O}, 0^{n}\right)$ and a large subset $S_{\vartheta} \subseteq 0^{k} 1 \Sigma^{n}$ such that $\operatorname{subtree}\left(N, \mathcal{O}, 0^{n}, \vartheta\right)$ is unambiguous and for every $\alpha \in S_{\vartheta}$, 
subtree $\left(N, \mathcal{O} \cup\{\alpha\}, 0^{n}, \vartheta\right)$ accepts with unambiguity if and only if $\operatorname{subtree}\left(N, \mathcal{O}, 0^{n}, \vartheta\right)$ rejects with unambiguity. Because of this nice relationship that preserves unambiguity, we can focus on the subtree rooted at $\vartheta$, instead of the computation tree of $N^{\mathcal{O}}\left(0^{n}\right)$, at the cost of limiting to $S_{\vartheta}$ for extending the partial oracle $\mathcal{O}$ with strings in $0^{k} 1 \Sigma^{n}$.

Lemma 4.7 Let $k, n \in \mathbb{N}^{+}$. Let $p$ be a polynomial and let $N$ be a polynomial-time bounded normalized ATM such that, for every oracle $B \subseteq \Sigma^{*}, N^{B}$ is a k-level ATM and $p($.$) is the switch time of N^{B}$. Let $\mathcal{O}$ be an arbitrary partial oracle such that $\mathcal{O} \cap 0^{k} 1 \Sigma^{n}=$ $\emptyset$. Assume that for every $\alpha \in 0^{k} 1 \Sigma^{n}, N^{\mathcal{O} \cup\{\alpha\}}\left(0^{n}\right)$ accepts with unambiguity, whereas $N^{\mathcal{O}}\left(0^{n}\right)$ rejects with unambiguity or loses unambiguity somewhere in its computation tree. Then, there is a salient node $\vartheta$ in $\operatorname{ctree}\left(N, \mathcal{O}, 0^{n}\right)$ and a set $S_{\vartheta} \subseteq 0^{k} 1 \Sigma^{n}$ with $\left\|S_{\vartheta}\right\| \geq 2^{n-1}-\frac{k \cdot p(n)}{2}$ such that the following hold:

1. For every $S^{\prime} \subseteq S_{\vartheta}, \vartheta$ is a salient node at a fixed (independent of $S^{\prime}$ ) level $\leq k$ in ctree $\left(N, \mathcal{O} \cup \bar{S}^{\prime}, 0^{n}\right)$.

2. If $\vartheta$ is an existential node, then (a) subtree $\left(N, \mathcal{O}, 0^{n}, \vartheta\right)$ rejects with unambiguity, and (b) for each $\alpha \in S_{\vartheta}$, subtree $\left(N, \mathcal{O} \cup\{\alpha\}, 0^{n}, \vartheta\right)$ accepts with unambiguity. (Here, we rely on (1) that for any $S^{\prime} \subseteq S_{\vartheta}, \vartheta$ is a salient node in ctree $(N, \mathcal{O} \cup$ $\left.S^{\prime}, 0^{n}\right)$.)

3. If $\vartheta$ is a universal node, then (a) subtree $\left(N, \mathcal{O}, 0^{n}, \vartheta\right)$ accepts with unambiguity, and (b) for each $\alpha \in S_{\vartheta}$, subtree $\left(N, \mathcal{O} \cup\{\alpha\}, 0^{n}, \vartheta\right)$ rejects with unambiguity. (Here again, we rely on (1) that for any $S^{\prime} \subseteq S_{\vartheta}, \vartheta$ is a salient node in ctree $(N, \mathcal{O} \cup$ $\left.S^{\prime}, 0^{n}\right)$.)

Proof Let $\varphi$ denote the root of $\operatorname{ctree}\left(N, \mathcal{O}, 0^{n}\right)$. If $N^{\mathcal{O}}\left(0^{n}\right)$ rejects with unambiguity, then clearly $\vartheta:=\varphi$ and $S_{\vartheta}:=0^{k} 1 \Sigma^{n}$ suffice for the proof (since, by the assumption made in Section 2, the root of any oracle ATM is an existential node). Henceforward, assume that $N^{\mathcal{O}}\left(0^{n}\right)$ loses unambiguity somewhere in its computation tree. Let $r={ }_{d f} \max _{1 \leq \ell \leq k}\left\{\ell \mid\right.$ there is a salient node $\zeta_{\ell}$ at level $\ell$ in $\operatorname{ctree}\left(N, \mathcal{O}, 0^{n}\right)$ such that $\operatorname{subtree}\left(N, \mathcal{\mathcal { O }}, 0^{n}, \zeta_{\ell}\right)$ loses unambiguity\}. W.l.o.g., we assume that $\zeta_{r}$ is an existential node; an almost similar argument can be given when $\zeta_{r}$ is a universal node. Let $\gamma_{1}$ and $\gamma_{2}$ be the nodes at level $r+1$ such that the paths from $\zeta_{r}$ to $\gamma_{1}$ and from $\zeta_{r}$ to $\gamma_{2}$ in $\operatorname{ctree}\left(N, \mathcal{O}, 0^{n}\right)$ are accepting. Let $\rho_{\varphi \rightarrow \gamma_{1}}$ and $\rho_{\varphi \rightarrow \gamma_{2}}$ be the paths from $\varphi$ to $\gamma_{1}$ and from $\varphi$ to $\gamma_{2}$, respectively, in ctree $\left(N, \mathcal{O}, 0^{n}\right)$. Define a set $U$ consisting of all strings in $0^{k} 1 \Sigma^{n}$ not queried along $\rho_{\varphi \rightarrow \gamma_{1}}$ and $\rho_{\varphi \rightarrow \gamma_{2}}$. (It is easy to see that $\|U\| \geq 2^{n}-(r+1) \cdot p(n)$.) By our choice of $U$, for any $U^{\prime} \subseteq U, \zeta_{r}, \gamma_{1}$, and $\gamma_{2}$ are nodes in ctree $\left(N, \mathcal{O} \cup U^{\prime}, 0^{n}\right)$. If $r=k$, then for any $\alpha \in U, N^{\mathcal{O} \cup\{\alpha\}}\left(0^{n}\right)$ loses unambiguity, since the paths from $\zeta_{r}$ to $\gamma_{1}$ and from $\zeta_{r}$ to $\gamma_{2}$ are accepting in $\operatorname{ctree}\left(N, \mathcal{O} \cup\{\alpha\}, 0^{n}\right)$. This contradicts the assumption that, for any $\alpha \in 0^{k} 1 \Sigma^{n}, N^{\mathcal{O} \cup\{\alpha\}}\left(0^{n}\right)$ accepts with unambiguity. So, we now assume that $r<k$. This assumption implies that any computation subtree rooted at $\gamma_{1}$ or at $\gamma_{2}$ is well-defined (i.e., has depth at least 1). If for some $\alpha \in U$, subtree $\left(N, \mathcal{O} \cup\{\alpha\}, 0^{n}, \gamma_{1}\right)$ and subtree $\left(N, \mathcal{O} \cup\{\alpha\}, 0^{n}, \gamma_{2}\right)$ are both accepting, then $N^{\mathcal{O} \cup\{\alpha\}}\left(0^{n}\right)$ loses unambiguity; this contradicts the assumption that, for every $\alpha \in 0^{k} 1 \Sigma^{n}, N^{\mathcal{O} \cup\{\alpha\}}\left(0^{n}\right)$ accepts with unambiguity. Thus, for every $\alpha \in U$, at least one of subtree $\left(N, \mathcal{O} \cup\{\alpha\}, 0^{n}, \gamma_{1}\right)$ and subtree $\left(N, \mathcal{O} \cup\{\alpha\}, 0^{n}, \gamma_{2}\right)$ is rejecting. By averaging principle, there is some $\nu \in\left\{\gamma_{1}, \gamma_{2}\right\}$ such that, for at least $\|U\| / 2$ strings $\alpha \in U$, subtree $\left(N, \mathcal{O} \cup\{\alpha\}, 0^{n}, \nu\right)$ is rejecting and let $U^{\prime} \subseteq U$ be the set of $\alpha \in U$ such that $\operatorname{subtree}\left(N, \mathcal{O} \cup\{\alpha\}, 0^{n}, \nu\right)$ rejects. Clearly, $\left\|U^{\prime}\right\| \geq 2^{n-1}-\frac{(r+1) \cdot p(n)}{2} \geq 2^{n-1}-\frac{k \cdot p(n)}{2}$. 
Note that $\nu$ is a universal node. The lemma follows by setting $\vartheta:=\nu$ and $S_{\vartheta}:=U^{\prime}$.

For any sentinel node $\vartheta$ in a computation tree and string $\alpha$, we introduce notations $s(\vartheta, \alpha)$ and $Q_{\vartheta \rightarrow s(\vartheta, \alpha)}$ that will be used in the proof of Lemma 4.9 and 4.10.

Notation 4.8 Let $k, n \in \mathbb{N}^{+}$. Let $N$ be a polynomial-time bounded normalized ATM such that, for every oracle $B \subseteq \Sigma^{*}, N^{B}$ is a k-level ATM with a fixed (independent of $B$ ) switch time. Let $\mathcal{O}_{1}$ and $\mathcal{O}_{2}$ be arbitrary partial oracles such that $\mathcal{O}_{1} \cap 0^{k} 1 \Sigma^{n}=\emptyset$ and $\mathcal{O}_{2} \subseteq 0^{k} 1 \Sigma^{n}$. Let $\vartheta$ be an existential (universal) sentinel node in ctree $\left(N, \mathcal{O}_{1}, 0^{n}\right)$ and let $S_{\vartheta} \subseteq 0^{k} 1 \Sigma^{n}-\mathcal{O}_{2}$ be such that (a) for every $S^{\prime} \subseteq S_{\vartheta}, \vartheta$ is a sentinel node at a fixed (independent of $S^{\prime}$ ) level $<k$ in $\operatorname{ctree}\left(N, \mathcal{O}_{1} \cup \mathcal{O}_{2} \cup S^{\prime}, 0^{n}\right)$, (b) subtree $\left(N, \mathcal{O}_{1} \cup \mathcal{O}_{2}, 0^{n}, \vartheta\right)$ rejects (respectively, accepts) with unambiguity, and (c) for every $\alpha \in S_{\vartheta}$, subtree $\left(N, \mathcal{O}_{1} \cup \mathcal{O}_{2} \cup\{\alpha\}, 0^{n}, \vartheta\right)$ accepts (respectively, rejects) with unambiguity. Then, for every $\alpha \in S_{\vartheta}, s(\vartheta, \alpha)$ denotes the unique universal (respectively, existential) sentinel node such that the path from $\vartheta$ to $s(\vartheta, \alpha)$ is accepting (respectively, rejecting) and level $(s(\vartheta, \alpha))=\operatorname{level}(\vartheta)+1$ in $\operatorname{ctree}\left(N, \mathcal{O}_{1} \cup \mathcal{O}_{2} \cup\{\alpha\}, 0^{n}\right)$. In the same context, we use $Q_{\vartheta \rightarrow s(\vartheta, \alpha)}$ to denote the set of queries along the path from $\vartheta$ to $s(\vartheta, \alpha)$ in $\operatorname{ctree}\left(N, \mathcal{O}_{1} \cup \mathcal{O}_{2} \cup\{\alpha\}, 0^{n}\right)$.

The proof of Theorem 4.4 uses inductive argument on the level of a salient node $\vartheta$ in a computation tree. Lemma 4.9 states the base step of the inductive argument.

Lemma 4.9 (Base Step) Let $k, n \in \mathbb{N}^{+}$. Let $p$ be a polynomial and let $N$ be a polynomial-time bounded normalized ATM such that, for every oracle $B \subseteq \Sigma^{*}, N^{B}$ is a k-level ATM and $p($.$) is the switch time of N^{B}$. Let $\mathcal{O}_{1}$ and $\mathcal{O}_{2}$ be arbitrary partial oracles such that $\mathcal{O}_{1} \cap 0^{k} 1 \Sigma^{n}=\emptyset, \mathcal{O}_{2} \subseteq 0^{k} 1 \Sigma^{n}$, and $1 \leq\left\|\mathcal{O}_{2}\right\| \leq k-1$. Let $\vartheta$ be an existential (universal) sentinel node in ctree $\left(N, \mathcal{O}_{1} \cup \mathcal{O}_{2}, 0^{n}\right)$ and let $S_{\vartheta} \subseteq 0^{k} 1 \Sigma^{n}-\mathcal{O}_{2}$ be a set such that, for every $S^{\prime} \subseteq S_{\vartheta}, \vartheta$ is a salient node at the $k^{\prime}$ th level, i.e., $\operatorname{level}(\vartheta)=k$, in $\operatorname{ctree}\left(N, \mathcal{O}_{1} \cup \mathcal{O}_{2} \cup S^{\prime}, 0^{n}\right)$ and $\left\|S_{\vartheta}\right\|>2 p(n)+1$. Assume that subtree $\left(N, \mathcal{O}_{1} \cup \mathcal{O}_{2}, 0^{n}, \vartheta\right)$ rejects (respectively, accepts), while for every $\alpha \in S_{\vartheta}$, subtree $\left(N, \mathcal{O}_{1} \cup \mathcal{O}_{2} \cup\{\alpha\}, 0^{n}, \vartheta\right)$ accepts (respectively, rejects) with unambiguity. Then, there exist $\alpha, \beta \in S_{\vartheta}$ such that subtree $\left(N, \mathcal{O}_{1} \cup \mathcal{O}_{2} \cup\{\alpha, \beta\}, 0^{n}, \vartheta\right)$ is ambiguous.

Proof The proof is similar to that of Theorem 3.1.1(b) in [CGH ${ }^{+}$89]. W.l.o.g., assume that $\vartheta$ is an existential node in $\operatorname{ctree}\left(N, \mathcal{O}_{1} \cup \mathcal{O}_{2}, 0^{n}\right)$. Note that the assumptions in Lemma 4.9 imply that, for every $\alpha \in S_{\vartheta}, Q_{\vartheta \rightarrow s(\vartheta, \alpha)} \neq \emptyset$. Using the Party Lemma (i.e., Lemma 4.6) with parameters $k=2$ and $\ell=\left\|S_{\vartheta}\right\|$, there exists $T \subseteq S_{\vartheta}$ with $\|T\|=2$ such that, for all $\alpha \in T, Q_{\vartheta \rightarrow s(\vartheta, \alpha)} \cap(T-\{\alpha\})=\emptyset$. It follows that subtree $\left(N, \mathcal{O}_{1} \cup \mathcal{O}_{2} \cup T, 0^{n}, \vartheta\right)$ has two accepting paths.

Lemma 4.10 is the inductive step in the proof of Theorem 4.4. Informally, Lemma 4.10 states that if $N$ is a polynomial-time bounded normalized ATM such that, for every oracle $B \subseteq \Sigma^{*}, N^{B}$ is a $k$-level ATM with a fixed switch time, if $\mathcal{O}_{1}$ and $\mathcal{O}_{2}$ are arbitrary partial oracles such that $\mathcal{O}_{1} \cap 0^{k} 1 \Sigma^{n}=\emptyset$ and $\mathcal{O}_{2} \subseteq 0^{k} 1 \Sigma^{n}$, if $\vartheta$ is an existential (universal) sentinel node in the computation tree of $N^{\mathcal{O}_{1} \cup \mathcal{O}_{2}}\left(0^{n}\right)$, and if $S_{\vartheta} \subseteq 0^{k} 1 \Sigma^{n}-\mathcal{O}_{2}$ is a large set, then the following is true: If $\operatorname{subtree}\left(N, \mathcal{O}_{1} \cup \mathcal{O}_{2}, 0^{n}, \vartheta\right)$ rejects (respectively, accepts) with unambiguity, if for every $\alpha \in S_{\vartheta}$, subtree $\left(N, \mathcal{O}_{1} \cup \mathcal{O}_{2} \cup\{\alpha\}, 0^{n}, \vartheta\right)$ accepts (respectively, rejects) with unambiguity, and if for every $\alpha_{1}, \alpha_{2} \in S_{\vartheta}$, $\operatorname{subtree}\left(N, \mathcal{O}_{1} \cup\right.$ $\left.\mathcal{O}_{2} \cup\left\{\alpha_{1}, \alpha_{2}\right\}, 0^{n}, \vartheta\right)$ is unambiguous, then there is a sentinel node $\eta$ in subtree $\left(N, \mathcal{O}_{1} \cup\right.$ 
$\left.\mathcal{O}_{2}, 0^{n}, \vartheta\right)$, a set $T_{\eta} \subseteq S_{\vartheta}$ containing at most one element, and a large subset $S_{\eta} \subseteq$ $S_{\vartheta}-T_{\eta}$ such that, for every $\alpha \in S_{\eta}$, subtree $\left(N, \mathcal{O}_{1} \cup \mathcal{O}_{2} \cup T_{\eta} \cup\{\alpha\}, 0^{n}, \eta\right)$ accepts with unambiguity if and only if subtree $\left(N, \mathcal{O}_{1} \cup \mathcal{O}_{2} \cup T_{\eta}, 0^{n}, \eta\right)$ rejects with unambiguity. Because of this nice relationship that preserves unambiguity, we can focus on the subtree rooted at $\eta$, instead of the subtree rooted at $\vartheta$, at the cost of extending the partial oracle $\mathcal{O}_{1} \cup \mathcal{O}_{2}$ to $\mathcal{O}_{1} \cup \mathcal{O}_{2} \cup T_{\eta}$ and limiting to $S_{\eta}$ for extension of the partial oracle $\mathcal{O}_{1} \cup \mathcal{O}_{2} \cup T_{\eta}$ with strings in $0^{k} 1 \Sigma^{n}$ at later steps.

Lemma 4.10 (Inductive Step) Let $k, n \in \mathbb{N}^{+}$and let $\ell=\max \{0, k-2\}$. Let $p$ be a polynomial and let $N$ be a polynomial-time bounded normalized ATM such that, for every oracle $B \subseteq \Sigma^{*}, N^{B}$ is a $k$-level $A T M$ and $p($.$) is the switch time of N^{B}$. Let $\mathcal{O}_{1}$ and $\mathcal{O}_{2}$ be arbitrary partial oracles such that $\mathcal{O}_{1} \cap 0^{k} 1 \Sigma^{n}=\emptyset, \mathcal{O}_{2} \subseteq 0^{k} 1 \Sigma^{n}$, and $\left\|\mathcal{O}_{2}\right\|=\ell$. Let $\vartheta$ be an existential (universal) node in ctree $\left(N, \mathcal{O}_{1} \cup \mathcal{O}_{2}, 0^{n}\right)$ and let $S_{\vartheta} \subseteq 0^{k} 1 \Sigma^{n}-\mathcal{O}_{2}$ be a set such that, for every $S^{\prime} \subseteq S_{\vartheta}, \vartheta$ is a salient node at a fixed (independent of $S^{\prime}$ ) level $<k$ in $\operatorname{ctree}\left(N, \mathcal{O}_{1} \cup \mathcal{O}_{2} \cup S^{\prime}, 0^{n}\right)$ and $\left\|S_{\vartheta}\right\| \gg 64 p^{2}(n)$. Assume that (i) subtree $\left(N, \mathcal{O}_{1} \cup \mathcal{O}_{2}, 0^{n}, \vartheta\right)$ rejects (respectively, accepts) with unambiguity, (ii) for every $\alpha \in S_{\vartheta}$, subtree $\left(N, \mathcal{O}_{1} \cup \mathcal{O}_{2} \cup\{\alpha\}, 0^{n}, \vartheta\right)$ accepts (respectively, rejects) with unambiguity, and (iii) for every $\alpha_{1}, \alpha_{2} \in S_{\vartheta}$, $\operatorname{subtree}\left(N, \mathcal{O}_{1} \cup \mathcal{O}_{2} \cup\left\{\alpha_{1}, \alpha_{2}\right\}, 0^{n}, \vartheta\right)$ is unambiguous. Then, there is a sentinel node $\eta$ in $\operatorname{subtree}\left(N, \mathcal{O}_{1} \cup \mathcal{O}_{2}, 0^{n}, \vartheta\right)$, a set $T_{\eta} \subseteq S_{\vartheta}$ with $0 \leq\left\|T_{\eta}\right\| \leq 1$, and a set $S_{\eta} \subseteq S_{\vartheta}-T_{\eta}$ such that the following hold:

1. For every $S^{\prime} \subseteq S_{\eta}, \eta$ is a salient node at a fixed (independent of $S^{\prime}$ ) level $\leq k$, where level $(\vartheta)+1 \leq \operatorname{level}(\eta) \leq \operatorname{level}(\vartheta)+2$, in $\operatorname{ctree}\left(N, \mathcal{O}_{1} \cup \mathcal{O}_{2} \cup S^{\prime}, 0^{n}\right)$.

2. If level $(\eta)=\operatorname{level}(\vartheta)+2$, then (a) $T_{\eta}=\emptyset$, (b) subtree $\left(N, \mathcal{O}_{1} \cup \mathcal{O}_{2} \cup T_{\eta}, 0^{n}, \eta\right)$ rejects (respectively, accepts) with unambiguity, and (c) for each $\alpha \in S_{\eta}$, subtree $\left(N, \mathcal{O}_{1} \cup\right.$ $\left.\mathcal{O}_{2} \cup T_{\eta} \cup\{\alpha\}, 0^{n}, \eta\right)$ accepts (respectively, rejects) with unambiguity.

3. If level $(\eta)=\operatorname{level}(\vartheta)+1$, then (a) $\left\|T_{\eta}\right\|=1$, (b) $\operatorname{subtree}\left(N, \mathcal{O}_{1} \cup \mathcal{O}_{2} \cup T_{\eta}, 0^{n}, \eta\right)$ accepts (respectively, rejects) with unambiguity, and (c) for each $\alpha \in S_{\eta}$, subtree $\left(N, \mathcal{O}_{1} \cup \mathcal{O}_{2} \cup T_{\eta} \cup\{\alpha\}, 0^{n}, \eta\right)$ rejects (respectively, accepts) with unambiguity.

4. $\left\|S_{\eta}\right\| \geq\left\|S_{\vartheta}\right\|^{1 / 4}$.

Proof W.l.o.g., assume that $\vartheta$ is an existential node in $\operatorname{ctree}\left(N, \mathcal{O}_{1} \cup \mathcal{O}_{2}, 0^{n}\right)$ with $\operatorname{level}(\vartheta)<k$. Let $U=_{d f}\left\{\alpha_{i} \in S_{\vartheta} \mid Q_{\vartheta \rightarrow s\left(\vartheta, \alpha_{i}\right)}=\emptyset\right\}$. We consider two cases.

Case 1: $\|\boldsymbol{U}\| \geq\left\|\boldsymbol{S}_{\boldsymbol{\vartheta}}\right\| / \mathbf{2}$. Define an equivalence relation $\varrho$ on $U$ as follows: $\forall \alpha_{i}, \alpha_{j} \in$ $U$,

$$
\alpha_{i} \varrho \alpha_{j} \Longleftrightarrow s\left(\vartheta, \alpha_{i}\right)=s\left(\vartheta, \alpha_{j}\right)
$$

Case 1.a) There is an equivalence class of $\varrho$ of size $\geq\left\|S_{\vartheta}\right\|^{\mathbf{1 / 2}}$. Let this equivalence class be denote by $U^{\prime}$ and let $\alpha_{i}$ be an arbitrary element of $U^{\prime}$. Note that $\alpha_{i} \in U^{\prime} \subseteq U$ implies that $s\left(\vartheta, \alpha_{i}\right)$ is a node in ctree $\left(N, \mathcal{O}_{1} \cup \mathcal{O}_{2}, 0^{n}\right)$. Since subtree $\left(N, \widehat{\mathcal{O}_{1}} \cup \mathcal{O}_{2}, 0^{n}, s\left(\vartheta, \alpha_{i}\right)\right)$ rejects with unambiguity and since $s\left(\vartheta, \alpha_{i}\right)$ is a universal node, there is a unique rejecting path $\rho_{s\left(\vartheta, \alpha_{i}\right) \rightarrow \eta^{\prime}}$ from $s\left(\vartheta, \alpha_{i}\right)$ to a sentinel node or a leaf node $\eta^{\prime}$ in subtree $\left(N, \mathcal{O}_{1} \cup \mathcal{O}_{2}, 0^{n}, s\left(\vartheta, \alpha_{i}\right)\right)$. Let $Q_{\eta^{\prime}}$ denote the set of queries along $\rho_{s\left(\vartheta, \alpha_{i}\right) \rightarrow \eta^{\prime}}$ in $N^{\mathcal{O}_{1} \cup \mathcal{O}_{2}}\left(0^{n}\right)$ and let $T={ }_{d f} U^{\prime}-Q_{\eta^{\prime}}$. If $\eta^{\prime}$ is a leaf node, then for any $\alpha \in T$, subtree $\left(N, \mathcal{O}_{1} \cup \mathcal{O}_{2} \cup\right.$ $\left.\{\alpha\}, 0^{n}, s\left(\vartheta, \alpha_{i}\right)\right)$ is rejecting. This gives a contradiction with the assumption that, for any $\alpha \in U^{\prime}$, $\operatorname{subtree}\left(N, \mathcal{O}_{1} \cup \mathcal{O}_{2} \cup\{\alpha\}, 0^{n}, s\left(\vartheta, \alpha_{i}\right)\right)$ is accepting. 
Henceforward, we assume that $\eta^{\prime}$ is a sentinel node with level $\left(\eta^{\prime}\right) \leq k$. Then, the following hold: (a) for every $S^{\prime} \subseteq T, \eta^{\prime}$ is an existential sentinel node at level $(\vartheta)+2 \leq k$ in $\operatorname{ctree}\left(N, \mathcal{O}_{1} \cup \mathcal{O}_{2} \cup S^{\prime}, 0^{n}\right)$, (b) subtree $\left(N, \mathcal{O}_{1} \cup \mathcal{O}_{2}, 0^{n}, \eta^{\prime}\right)$ rejects with unambiguity, (c) for every $\alpha \in T$, subtree $\left(N, \mathcal{O}_{1} \cup \mathcal{O}_{2} \cup\{\alpha\}, 0^{n}, \eta^{\prime}\right)$ accepts with unambiguity, and (d) $\|T\| \geq\left\|S_{\vartheta}\right\|^{1 / 2}-p(n) \geq\left\|S_{\vartheta}\right\|^{1 / 4}$. The lemma follows by setting $\eta:=\eta^{\prime}, T_{\eta}:=\emptyset$, and $S_{\eta}:=T$.

Case 1.b) The size of any equivalence class of $\varrho$ is $<\left\|S_{\vartheta}\right\|^{1 / 2}$. Define a set $T$ consisting of exactly one element from each of the equivalence classes of $\varrho$ on $U$. Then, for any $\alpha_{1}, \alpha_{2} \in T, s\left(\vartheta, \alpha_{1}\right) \neq s\left(\vartheta, \alpha_{2}\right)$. Also, $\|T\| \geq\|U\| /\left\|S_{\vartheta}\right\|^{1 / 2} \geq\left\|S_{\vartheta}\right\|^{1 / 2} / 2$. If, for distinct $\alpha_{1}, \alpha_{2} \in T$, it holds that subtree $\left(N, \mathcal{O}_{1} \cup \mathcal{O}_{2} \cup\left\{\alpha_{1}, \alpha_{2}\right\}, 0^{n}, s\left(\vartheta, \alpha_{1}\right)\right)$ and subtree $\left(N, \mathcal{O}_{1} \cup\right.$ $\left.\mathcal{O}_{2} \cup\left\{\alpha_{1}, \alpha_{2}\right\}, 0^{n}, s\left(\vartheta, \alpha_{2}\right)\right)$ are both accepting, then subtree $\left(N, \mathcal{O}_{1} \cup \mathcal{O}_{2} \cup\right.$ $\left.\left\{\alpha_{1}, \alpha_{2}\right\}, 0^{n}, \vartheta\right)$ loses unambiguity; this contradicts our assumption that, for every $\alpha_{1}, \alpha_{2} \in S_{\vartheta}$, subtree $\left(N, \mathcal{O}_{1} \cup \mathcal{O}_{2} \cup\left\{\alpha_{1}, \alpha_{2}\right\}, 0^{n}, \vartheta\right)$ is unambiguous. So, we now assume that, for every $\alpha_{1}, \alpha_{2} \in T$, at least one of subtree $\left(N, \mathcal{O}_{1} \cup\right.$ $\left.\mathcal{O}_{2} \cup\left\{\alpha_{1}, \alpha_{2}\right\}, 0^{n}, s\left(\vartheta, \alpha_{1}\right)\right)$ and subtree $\left(N, \mathcal{O}_{1} \cup \mathcal{O}_{2} \cup\left\{\alpha_{1}, \alpha_{2}\right\}, 0^{n}, s\left(\vartheta, \alpha_{2}\right)\right)$ is rejecting. By averaging principle, there must be some $\beta \in T$ such that, for at least $\|T\| / 2 \alpha_{i}$ 's $\in T$, subtree $\left(N, \mathcal{O}_{1} \cup \mathcal{O}_{2} \cup\left\{\beta, \alpha_{i}\right\}, 0^{n}, s(\vartheta, \beta)\right)$ is rejecting. Let this majority set consisting of at least $\|T\| / 2$ elements of $T$ be denoted by $T^{\prime}$. Note that $\left\|T^{\prime}\right\| \geq\left\|S_{\vartheta}\right\|^{1 / 2} / 4 \geq\left\|S_{\vartheta}\right\|^{1 / 4}$. The lemma follows by setting $\eta:=s(\vartheta, \beta), T_{\eta}:=\{\beta\}$, and $S_{\eta}:=T^{\prime}$.

Case 2: $\|\boldsymbol{U}\|<\left\|\boldsymbol{S}_{\vartheta}\right\| / \mathbf{2}$. Let $U^{\prime}=_{d f} S_{\vartheta}-U$. Then, $\left\|U^{\prime}\right\| \geq\left\|S_{\vartheta}\right\| / 2$ and for any $\alpha_{i} \in U^{\prime}, Q_{\vartheta \rightarrow s\left(\vartheta, \alpha_{i}\right)} \neq \emptyset$. Apply the Party Lemma $\left[\mathrm{CGH}^{+} 89\right]$ with parameters $\ell=\left\|U^{\prime}\right\|$ and $k=2 \cdot\left\|S_{\vartheta}\right\|^{1 / 4}$. Since, for every $\alpha \in U^{\prime}, 1 \leq\left\|Q_{\vartheta \rightarrow s(\vartheta, \alpha)}\right\| \leq p(n)$ and $\left\|S_{\vartheta}\right\| \gg 64 \cdot p^{2}(n)$, therefore there exists $T \subseteq U^{\prime}$ such that $\|T\|=k$ and for all $\alpha \in T, Q_{\vartheta \rightarrow s(\vartheta, \alpha)} \cap(T-\{\alpha\})=\emptyset$. If for some distinct $\alpha_{1}, \alpha_{2} \in T$, it holds that subtree $\left(N, \mathcal{O}_{1} \cup \mathcal{O}_{2} \cup\left\{\alpha_{1}, \alpha_{2}\right\}, 0^{n}, s\left(\vartheta, \alpha_{1}\right)\right)$ and subtree $\left(N, \mathcal{O}_{1} \cup \mathcal{O}_{2} \cup\right.$ $\left.\left\{\alpha_{1}, \alpha_{2}\right\}, 0^{n}, s\left(\vartheta, \alpha_{2}\right)\right)$ are both accepting, then subtree $\left(N, \mathcal{O}_{1} \cup \mathcal{O}_{2} \cup\left\{\alpha_{1}, \alpha_{2}\right\}, 0^{n}, \vartheta\right)$ loses unambiguity; thus, contradicting our assumption. So, assume now that for every $\alpha_{1}, \alpha_{2} \in T$, at least one of subtree $\left(N, \mathcal{O}_{1} \cup \mathcal{O}_{2} \cup\left\{\alpha_{1}, \alpha_{2}\right\}, 0^{n}, s\left(\vartheta, \alpha_{1}\right)\right)$ and subtree $\left(N, \mathcal{O}_{1} \cup \mathcal{O}_{2} \cup\left\{\alpha_{1}, \alpha_{2}\right\}, 0^{n}, s\left(\vartheta, \alpha_{2}\right)\right)$ is rejecting. By averaging principle, there must be some $\beta \in T$ such that, for at least $\|T\| / 2 \alpha_{i}$ 's $\in T$, $\operatorname{subtree}\left(N, \mathcal{O}_{1} \cup\right.$ $\left.\mathcal{O}_{2} \cup\left\{\beta, \alpha_{i}\right\}, 0^{n}, s(\vartheta, \beta)\right)$ is rejecting. Let this majority set consisting of at least $\|T\| / 2$ elements of $T$ be denoted by $T^{\prime}$. The lemma follows by setting $\eta:=s(\vartheta, \beta)$, $T_{\eta}:=\{\beta\}$, and $S_{\eta}:=T^{\prime}$.

(Lemma 4.10)

\section{Power of Robustly Unambiguous Alternating Machines}

Hartmanis and Hemachandra [HH90] showed that robustly categorical nondeterministic polynomial-time Turing machines (i.e., NPTMs that for no oracle and no input have more than one accepting path) accept simple languages in the sense that, for every oracle $A$, the languages accepted by such machines are computable in $\mathrm{P}^{\mathrm{NP} \oplus A}$. Thus, if $\mathrm{P}=\mathrm{NP}$, then nondeterministic polynomial-time Turing machines satisfying robustly categorical property cannot separate $\mathrm{P}^{A}$ from $\mathrm{NP}^{A}$, for any oracle $A$. Theorem 5.1 
generalizes this result of Hartmanis and Hemachandra [HH90] and shows that, for every oracle $A$, robustly $k$-level unambiguous polynomial-time alternating Turing machines accept languages that are computable in $\mathrm{P}^{\Sigma_{k}^{p} \oplus A}$. Thus, similar to the case of robustly categorical NPTMs, if $\mathrm{P}=\mathrm{NP}$, then robustly $k$-level unambiguous polynomial-time alternating Turing machines cannot separate $\mathrm{P}^{\mathcal{A}}$ from $\Sigma_{k}^{p, A}$, and consequently cannot separate $\mathrm{P}^{A}$ from $\mathrm{NP}^{A}$.

Theorem 5.1 For all $k \in \mathbb{N}^{+}$, the following holds:

$(\forall \mathcal{A})\left[N^{\mathcal{A}}\right.$ is a $k$-level unambiguous polynomial-time $\left.\mathrm{ATM}\right] \Longrightarrow(\forall \mathcal{A})\left[L\left(N^{\mathcal{A}}\right) \in \mathrm{P}^{\Sigma_{k}^{p} \oplus \mathcal{A}}\right]$.

Proof The proof is by induction on $k$. The base case, $k=1$, follows from Theorem

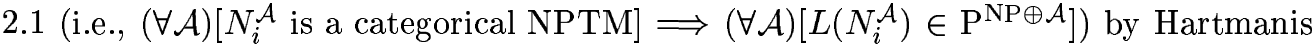
and Hemachandra [HH90]. Our induction hypothesis is the following: For all $j \leq k-1$, it holds that

$(\forall \mathcal{A})\left[N^{\mathcal{A}}\right.$ is a $j$-level unambiguous polynomial-time $\left.\mathrm{ATM}\right] \Longrightarrow(\forall \mathcal{A})\left[L\left(N^{\mathcal{A}}\right) \in \mathrm{P}^{\Sigma_{j}^{p} \oplus \mathcal{A}}\right]$.

Let $\mathcal{A}$ be an oracle and let $N$ be a robustly $k$-level unambiguous polynomial-time ATM. We define an oracle NPTM $\hat{N}$ with access to oracle $\Sigma_{k-1}^{p} \oplus \mathcal{A}$ as follows. On any input $x, \hat{N}^{\Sigma_{k-1}^{p} \oplus \mathcal{A}}$ guesses an existential computation path from the root (i.e., the level one node) to a universal node $\vartheta$ at level two in the computation tree of $N^{\mathcal{A}}(x)$. Upon reaching the node $\vartheta$ on this guessed path, $\hat{N}^{\Sigma_{k-1}^{p} \oplus \mathcal{A}}$ simulates the computation subtree of $N^{\mathcal{A}}(x)$ rooted at the node $\vartheta$. Since the computation subtree of $N^{\mathcal{A}}(x)$ rooted at the node $\vartheta$ is robustly $(k-1)$-level unambiguous, by induction hypothesis this simulation can be done in $\mathrm{P}^{\Sigma_{k-1}^{p} \oplus \mathcal{A}}$. Since $\mathcal{A}$ is arbitrary and $N$ is robustly $k$-level unambiguous polynomial-time ATM, $(\forall \mathcal{A})\left[L\left(\hat{N}^{\Sigma_{k-1}^{p} \oplus \mathcal{A}}\right)=L\left(N^{\mathcal{A}}\right)\right.$ and $\hat{N}^{\Sigma_{k-1}^{p} \oplus \mathcal{A}}$ is categorical $]$. We now show that $L\left(\hat{N}^{\Sigma_{k-1}^{p} \oplus \mathcal{A}}\right) \in \mathrm{P}^{\Sigma_{k}^{p} \oplus \mathcal{A}}$.

The proof of this part is the same as that of Theorem 2.1 by Hartmanis and Hemachandra [HH90]. Here, we give a sketch of the proof for the sake of completeness. Let $p($.$) be the running-time of \hat{N}$ with any oracle. We now define a polynomial-time computable procedure $M^{\Sigma_{k}^{p} \oplus \mathcal{A}}$ accepting $L\left(\hat{N}^{\Sigma_{k-1}^{p} \oplus \mathcal{A}}\right)$. On input $x, M^{\Sigma_{k}^{p} \oplus \mathcal{A}}(x)$ does the following:

1. Initialize database $S:=\emptyset$.

2. Repeat the following for $p(|x|)$ iterations:

Find an accepting path $\rho$ in the computation tree of $\hat{N}^{\Sigma_{k-1}^{p} \oplus \star}(x)$ consistent with $S$. (This step can be done in $\mathrm{P}^{\Sigma_{k}^{p}}$.) If no such $\rho$ exists, then halt and reject. Otherwise, i.e., if $\rho$ exists, then query $\mathcal{A}$ about the membership of strings queried along $\rho$ and update $S$ with this information. If the answers of queries along $\rho$ are consistent with $\mathcal{A}$, then halt and accept.

3. Accept if there is an accepting path in the computation tree of $\hat{N}^{\Sigma_{k-1}^{p} \oplus \star}(x)$ consistent with $S$ and consisting of queries none other than those in $S$, and reject if no such path exists.

Clearly, $M^{\Sigma_{k}^{p} \oplus \mathcal{A}}$ is computable in polynomial time. We now show that $M^{\Sigma_{k}^{p} \oplus \mathcal{A}}$ accepts $L\left(\hat{N}^{\Sigma_{k-1}^{P} \oplus \mathcal{A}}\right)$. By the definition of $M$, we only need to show that if $x \in L\left(\hat{N}^{\Sigma_{k-1}^{p} \oplus \mathcal{A}}\right)$, 
then $M^{\Sigma_{k}^{p} \oplus \mathcal{A}}(x)$ accepts. Suppose that for some input $x, \hat{N}^{\Sigma_{k-1}^{p} \oplus \mathcal{A}}(x)$ accepts. Let $T$ be the set of strings queried of $\mathcal{A}$ along the unique accepting computation path $\rho_{T}$ of $\hat{N}^{\Sigma_{k-1}^{p} \oplus \mathcal{A}}(x)$. If $M^{\Sigma_{k}^{p} \oplus \mathcal{A}}(x)$ accepts in some iteration of step 2, then we are done. So assume that $M^{\Sigma_{k}^{p} \oplus \mathcal{A}}(x)$ does not accept in any of the $p(|x|)$ iterations of step 2. This also implies that $M^{\Sigma_{k}^{p} \oplus \mathcal{A}}(x)$ does not reject in any iteration of step 2, since the accepting path $\rho_{T}$ has not been considered so far. Let $S_{i}$ be the set of strings queried of $\mathcal{A}$ in the $i^{\prime}$ th iteration of step 2 and let $\rho_{S_{i}}$ be the path found in this iteration. Then, there must be a query $q_{i}$ in $S_{i} \cap T$ answered in a conflicting way in $\rho_{S_{i}}$ and $\rho_{T}$; for if there is no such string, then there will be an oracle $\mathcal{O}$ consistent with the way strings in $S_{i} \cup T$ are answered; then relative to $\Sigma_{k-1}^{p} \oplus \mathcal{O}, \hat{N}(x)$ will have more than one accepting path; this will give a contradiction with our assumption that $(\forall \mathcal{A})\left[\hat{N}^{\Sigma_{k-1}^{p} \oplus \mathcal{A}}\right.$ is categorical $]$. This query $q_{i}$ must be different from $q_{j}$, for any $1 \leq j<i$, because the database $S$ in the $i$ 'th iteration of step 2 is consistent with $\mathcal{A}$ in the membership of any string queried in previous iterations. Thus, in each iteration of step 2, the membership in $\mathcal{A}$ of a new query from $T$ is found. So, after $p(|x|)$ iterations of step 2, the membership of all the strings queried along $\rho_{T}$ are known. It follows that $M^{\Sigma_{k}^{p} \oplus \mathcal{A}}(x)$ will accept on the execution of step 3 .

Corollary 5.2 For all $k \in \mathbb{N}^{+}$, if $\mathrm{P}=\mathrm{NP}$ and $(\forall \mathcal{A})\left[N^{\mathcal{A}}\right.$ is a $k$-level unambiguous polynomial-time ATM $]$ then $(\forall \mathcal{A})\left[L\left(N^{\mathcal{A}}\right) \in \mathrm{P}^{\mathcal{A}}\right]$.

Crescenzi and Silvestri [CS98] showed that languages accepted by robustly complementary and categorical oracle NPTMs are computable in $\mathrm{P}^{(\mathrm{UP} \cup \operatorname{coUP}) \oplus \mathcal{A}}$. In fact, their proof actually shows that the languages of such machines are computable in $\mathrm{P}^{\mathrm{UP} \oplus \mathcal{A}}$. Theorem 5.3 is a generalization of this result of Crescenzi and Silvestri [CS98] for robustly bounded-level unambiguous polynomial-time alternating Turing machines.

Theorem 5.3 For all $k_{i}, k_{j} \in \mathbb{N}^{+}$, the following holds: If, for all oracles $\mathcal{A}, N_{i}^{\mathcal{A}}$ and $N_{j}^{\mathcal{A}}$ are, respectively, $k_{i}$-level and $k_{j}$-level unambiguous polynomial-time ATMs and $L\left(N_{i}^{\mathcal{A}}\right)=\overline{L\left(N_{j}^{\mathcal{A}}\right)}$, then for all oracles $\mathcal{A}, L\left(N_{i}^{\mathcal{A}}\right) \in \mathrm{P}^{\mathrm{UP}^{\Sigma_{k-1}^{p}} \oplus \mathcal{A}}$, where $k=\max \left\{k_{i}, k_{j}\right\}$.

Proof Let $\mathcal{A}$ be an oracle and let $N_{i}, N_{j}$ be ATMs as in the statement of the theorem. Define oracle NPTMs $\hat{N}_{i}$ and $\hat{N}_{j}$ corresponding to $N_{i}$ and $N_{j}$, respectively, in the manner $\hat{N}$ is defined from an ATM $N$ in Theorem 5.1. Let $k=_{d f} \max \left\{k_{i}, k_{j}\right\}$. Thus, the following hold: (a) $(\forall \ell \in\{i, j\})(\forall \mathcal{A})\left[L\left(\hat{N}_{\ell}^{\Sigma_{k-1}^{p} \oplus \mathcal{A}}\right)=L\left(N_{\ell}^{\mathcal{A}}\right)\right.$ and $\hat{N}_{\ell}^{\Sigma_{k-1}^{p} \oplus \mathcal{A}}$

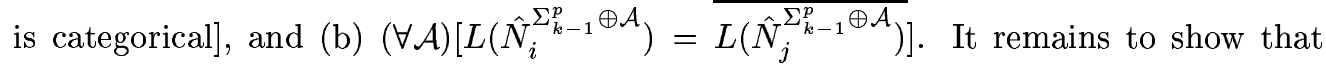
$L\left(\hat{N}_{i}^{\Sigma_{k-1}^{p} \oplus \mathcal{A}}\right) \in \mathrm{P}^{\mathrm{UP}^{\Sigma_{k-1}^{p}}}$. The proof of this part is omitted as it is identical to that of Theorem 8 (i.e., if $N_{0}$ and $N_{1}$ are two robustly complementary and categorical oracle NPTMs, then for all oracles $\left.\mathcal{A}, L\left(N_{0}^{\mathcal{A}}\right) \in \mathrm{P}^{(\mathrm{UP} \cup \operatorname{coUP}) \oplus \mathcal{A}}\right)$ by Crescenzi and Silvestri [CS98] and Theorem 2.1 by Hartmanis and Hemachandra [HH90].

\section{Open Questions}

We now mention some open questions and directions for further research. Theorem 3.5 implies that there is a relativized world where the unambiguity based hierarchies are infinite. However, a number of questions related to the relativized structure of 
unambiguity based hierarchies remain open. For instance, is there a relativized world where AUPH is finite, but UPH and $\mathcal{U P H}$ are infinite? Is there a relativized world where the polynomial hierarchy is infinite, but AUPH and UPH collapse?

Hemaspaandra and Rothe [HR97] showed that if UP has a sparse Turing-complete set, then for every $k \geq 3, \mathrm{U} \Sigma_{k}^{p} \subseteq \mathcal{U} \Sigma_{k-1}^{p}$. Are there other complexity-theoretic assumptions that can help in concluding about the structure of unambiguity based hierarchies?

Fortnow [For99] showed that $\mathrm{PH} \subset \mathrm{SPP}$ relative to a random oracle. Theorem 3.11 shows that there is a relativized world where UAP $\nsubseteq \mathrm{PH}$. Can we extend the oracle separation of UAP from $\mathrm{PH}$ to a random oracle separation?

Aida et al. [ACRW04] and Crâsmaru et al. [CGRS04] discussed whether UAP equals SPP. In fact, Crâsmaru et al. [CGRS04] pointed out their difficulty in building an oracle $\mathcal{A}$ such that $\mathrm{UAP}^{\mathcal{A}} \neq \mathrm{SPP}^{\mathcal{A}}$. Can the ideas involved in oracle constructions in this paper be used to attack this problem?

Finally, is it the case that similar to robustly bounded-level unambiguous polynomial-time ATMs, robustly unbounded-level unambiguous polynomial-time ATMs require weak oracle access in every relativized world?

Acknowledgment We thank Lane Hemaspaandra and Jörg Rothe for their helpful advice, guidance, and support.

\section{References}

[ACRW04] S. Aida, M. Crâsmaru, K. Regan, and O. Watanabe. Games with uniqueness properties. Theory of Computing Systems, 37(1):29-47, 2004.

[AK02] V. Arvind and P. Kurur. Graph isomorphism is in SPP. In Proceedings of the 43rd IEEE Symposium on Foundations of Computer Science, pages 743-750, Los Alamitos, November 16-19 2002. IEEE Computer Society.

[Bei89] R. Beigel. On the relativized power of additional accepting paths. In Proceedings of the 4th Structure in Complexity Theory Conference, pages 216-224. IEEE Computer Society Press, June 1989.

[BG82] A. Blass and Y. Gurevich. On the unique satisfiability problem. Information and Control, 55(1-3):80-88, 1982.

$\left[\mathrm{CGH}^{+} 89\right]$ J. Cai, T. Gundermann, J. Hartmanis, L. Hemachandra, V. Sewelson, K. Wagner, and G. Wechsung. The boolean hierarchy II: Applications. SIAM Journal on Computing, 18(1):95-111, 1989.

[CGRS04] M. Crâsmaru, C. Glaßer, K. Regan, and S. Sengupta. A protocol for serializing unique strategies. In Proceedings of the 29th International Symposium on Mathematical Foundations of Computer Science. SpringerVerlag Lecture Notes in Computer Science \#3153, August 2004.

[CKS81] A. Chandra, D. Kozen, and L. Stockmeyer. Alternation. Journal of the ACM, 26(1), 1981.

[CS98] P. Crescenzi and R. Silvestri. Sperner's lemma and robust machines. Computational Complexity, 7:163-173, 1998.

[For99] L. Fortnow. Relativized worlds with an infinite hierarchy. Information Processing Letters, 69(6):309-313, 1999. 
[GS88] J. Grollmann and A. Selman. Complexity measures for public-key cryptosystems. SIAM Journal on Computing, 17(2):309-335, 1988.

[Hås87] J. Håstad. Computational Limitations of Small-Depth Circuits. MIT Press, 1987.

[HH90] J. Hartmanis and L. Hemachandra. Robust machines accept easy sets. Theoretical Computer Science, 74(2):217-226, 1990.

[HR97] L. Hemaspaandra and J. Rothe. Unambiguous computation: Boolean hierarchies and sparse Turing-complete sets. SIAM Journal on Computing, 26(3):634-653, 1997.

[Ko85] K. Ko. On some natural complete operators. Theoretical Computer Science, $37(1): 1-30,1985$.

[Ko89] K. Ko. Relativized polynomial time hierarchies having exactly $k$ levels. SIAM Journal on Computing, 18(2):392-408, 1989.

[Ko91] K. Ko. Separating the low and high hierarchies by oracles. Information and Computation, 90(2):156-177, 1991.

[LR94] K.-J. Lange and P. Rossmanith. Unambiguous polynomial hierarchies and exponential size. In Proceedings of the 9th Structure in Complexity Theory Conference, pages 106-115. IEEE Computer Society Press, June/July 1994.

[NR98] R. Niedermeier and P. Rossmanith. Unambiguous computations and locally definable acceptance types. Theoretical Computer Science, 194(1-2):137$161,1998$.

[OH93] M. Ogiwara and L. Hemachandra. A complexity theory for feasible closure properties. Journal of Computer and System Sciences, 46(3):295-325, 1993.

[Pap94] C. Papadimitriou. Computational Complexity. Addison-Wesley, 1994.

[SL94] M. Sheu and T. Long. The extended low hierarchy is an infinite hierarchy. SIAM Journal on Computing, 23(3):488-509, 1994.

[SL96] M. Sheu and T. Long. UP and the low and high hierarchies: A relativized separation. Mathematical Systems Theory, 29(5):423-450, 1996.

[Sto77] L. Stockmeyer. The polynomial-time hierarchy. Theoretical Computer Science, 3:1-22, 1977.

[Wag92] K. Wagner. Alternating machines using partially defined "AND" and "OR". Technical Report 39, Institut für Informatik, Universität Würzburg, January 1992.

[Yao85] A. Yao. Separating the polynomial-time hierarchy by oracles. In Proceedings of the 26th IEEE Symposium on Foundations of Computer Science, pages $1-10,1985$. 